\title{
ON THE UPPER AND LOWER CLASS FOR STATIONARY GAUSSIAN PROCESSES $\left({ }^{1}\right)$
}

\author{
BY \\ TUNEKITI SIRAO $\left(^{2}\right)$ AND HISAO WATANABE $\left({ }^{3}\right)$
}

1. Introduction. Let $X=\{x(t) ; 0 \leqq t \leqq 1\}$ be a real stationary Gaussian process defined on a probability space $(\Omega, \mathscr{B}, P)$. As is well known, a stationary Gaussian process is uniquely determined by its mean value $m=E(x(t))$ and covariance function $\rho(h)=E((x(t+h)-m)(x(t)-m))$. Without loss of generality, we may assume that $m=0$ and $\rho(0)=1$. Then we have $\sigma^{2}(h)=2(1-\rho(h))$, where $\sigma^{2}(h)$ denotes $E\left((x(t+h)-x(t))^{2}\right)$, and accordingly all the information about $X$ is contained in $\sigma^{2}$.

Ju. K. Beljaev [1] has shown that for stationary Gaussian processes $X$ the following alternatives take place: either all sample functions are continuous, or all sample functions are unbounded in every interval of finite length (0-1 law). In the continuous case, he has further generalized G. A. Hunt's results [5] concerning Hölder continuity of sample functions. Before stating his result, we shall define the upper and lower classes for $X$. If there exists a positive number $\delta$ such that $0<|t-s|<\delta(0 \leqq t, s \leqq 1)$ implies $|f(t)-f(s)| \leqq g(|t-s|)$, then it is said that $f$ satisfies Lipschitz's condition relative to $g$.

Definition 1. Let $X=\{x(t) ; 0 \leqq t \leqq 1\}$ be a stationary Gaussian process. Then a monotone nondecreasing continuous function $\varphi$ defined on $[a, \infty)$ with $a>0$ is called a function belonging to the upper class (with respect to the uniform continuity of $X)$, if almost all sample functions $x(t, \omega)$ satisfy Lipschitz's condition relative to $g(h)=\sigma(h) \varphi\left(h^{-1}\right)$, i.e. for almost all $\omega$ there exists a $\delta(\omega)>0$ such that $0<|t-s|<\delta(\omega)$ implies

$$
|x(t, \omega)-x(s, \omega)| \leqq \sigma(t-s) \varphi(1 /|t-s|) .
$$

A monotone nondecreasing continuous function $\varphi$ is called a function belonging to the lower class (with respect to the uniform continuity of $X$ ), if almost all sample functions $x(t, \omega)$ do not satisfy Lipschitz's condition relative to $g(h)=\sigma(h) \varphi\left(h^{-1}\right)$, i.e. for almost all $\omega$ there exists a sequence $\left\{t_{n}(\omega) ; n=1,2,3, \ldots\right\} \subset[0,1]$ such that

$$
\left|x\left(t_{2 n}\right)-x\left(t_{2 n-1}\right)\right|>\sigma\left(\left|t_{2 n}-t_{2 n-1}\right|\right) \varphi\left(1 /\left|t_{2 n}-t_{2 n-1}\right|\right), \quad n \geqq 1,
$$

and $\left|t_{2 n}-t_{2 n-1}\right| \rightarrow 0$ as $n \rightarrow \infty$.

Received by the editors June 13, 1969.

( $\left.{ }^{1}\right)$ The results of this paper were partially reported in [8] without proof.

$\left({ }^{2}\right)$ Nagoya University and The Rockefeller University.

$\left({ }^{3}\right)$ Kyushu University.

Copyright (C) 1970, American Mathematical Society 
The collection of functions belonging to the upper class is denoted by $\mathscr{U}^{u}$ and the one for the lower class is denoted by $\mathscr{L}^{u}\left({ }^{4}\right)$.

Using these notations, Beljaev's result is stated as follows: Let $X$ be a continuous stationary Gaussian process with 0 -mean and assume that there exist positive constants $\delta, C_{1}<C_{2}$, and $\alpha(0<\alpha<2)$ such that for any $h \in(0, \delta)$

$$
C_{1} h^{\alpha} /|\log h| \leqq \sigma^{2}(h) \leqq C_{2} h^{\alpha} /|\log h|,
$$

and further $\sigma^{2}(h)$ is concave in $(0, \delta)$. Then the function $\varphi(t)=c\left\{\sigma\left(t^{-1}\right) t^{\alpha / 2}\right\}^{-1}$ belongs to $\mathscr{U}^{u}$ or $\mathscr{L}^{u}$ according as $c>\left(2 C_{2}\right)^{1 / 2} 2^{\alpha+1 / 2}\left(2^{\alpha / 2}-1\right)^{-1}$ or $c<\left(2 C_{1}\right)^{1 / 2}$. As is easily seen, we have unfortunately

$$
\left(2 C_{2}\right)^{1 / 2} \frac{2^{\alpha+1 / 2}}{2^{\alpha / 2}-1}>\left(2 C_{1}\right)^{1 / 2}, \quad 0<\alpha<2,0<C_{1}<C_{2} .
$$

On the other hand, the final form about the Hölder continuity of Wiener process is known which states: A monotone nondecreasing and continuous function $\varphi$ defined on $[a, \infty)$ belongs to $\mathscr{U}^{u}$ or $\mathscr{L}^{u}$ according as

$$
\int_{a}^{\infty} \varphi(t)^{3} \exp \left[-\frac{1}{2} \varphi^{2}(t)\right] d t<\infty \text { or }=\infty,
$$

(cf. Chung-Erdös-Sirao [3]). So we can see that

$$
\begin{gathered}
\varphi(t)=\left\{2 \log t+5 \log _{(2)} t+2 \log _{(3)} t+\cdots+2 \log _{(n-1)} t+(2+\varepsilon) \log _{(n)} t\right\}^{1 / 2}, \\
t \geqq e^{e^{. \cdot e} \quad(n-1 \text { times })}
\end{gathered}
$$

belongs to $\mathscr{U}^{u}$ or $\mathscr{L}^{u}$ according as $\varepsilon>0$ or $\varepsilon \leqq 0$, where $\log _{(k)} t$ denotes the $k$-fold iterated logarithm, i.e.

$$
\begin{array}{r}
\log _{(k)} t=\log \log \cdots \log t . \\
(k \text { times })
\end{array}
$$

Our main purpose in this paper is to give a criterion like (1), under certain conditions on $\sigma^{2}$, which decide if $\varphi$ belongs to $\mathscr{U}^{u}\left(\mathscr{L}^{u}\right)$ or $\operatorname{not}\left({ }^{5}\right)$.

The authors wish to express their hearty thanks to Professor K. Itô for his valuable suggestions.

2. Results. Throughout this paper, $X=\{x(t) ; 0 \leqq t \leqq 1\}$ is a real, continuous and stationary Gaussian process with zero mean, defined on the probability space $(\Omega, \mathscr{B}, P)$. We denote the correlation function of $X$ by $\rho$ and assume $\rho(0)=1$, and accordingly $\sigma^{2}(h)=2(1-\rho(h))$.

(4) The superscript $u$ expresses "with respect to the uniform continuity".

(5) $\mathscr{U}^{u}$ and $\mathscr{L}^{u}$ denote the collection of upper functions and lower functions defined for Wiener process, respectively. 
Let $g$ be a positive continuous function defined on $(0,1)$. For $a \geqq 0$, define $\mathscr{E}(a)$ by

$$
\mathscr{E}(a)=\left\{\omega ; \lim _{h \downarrow 0} \sup \left[\frac{x(t, \omega)-x(s, \omega)}{g(|t-s|)} ; 0 \leqq s, t \leqq 1,0<|t-s| \leqq h\right] \leqq a\right\}
$$

Then we have

THEOREM 1. If $\sigma(h) / g(h)$ tends to zero with $h$, then it follows that for any $a>0$, $P(\mathscr{E}(a))=0$ or 1 .

From this theorem, we can easily see the following

COROLLARY 1.1. If $\sigma(h) / g(h)$ tends to zero with $h$, then for any $a \geqq 0$

$$
P\left(\lim _{h \downarrow 0} \sup \left[\frac{x(t)-x(s)}{g(|t-s|)} ; 0 \leqq s, t \leqq 1,0<|t-s| \leqq h\right]=a\right)=1 \quad \text { or } \quad 0 .
$$

REMARK 1. The above corollary suggests that under Beljaev's assumption mentioned in $\S 1$, there exists a constant $C_{0}$ between $C_{1}$ and $C_{2}$ such that $\varphi(t)=$ $c\left\{t^{\alpha / 2} \sigma\left(t^{-1}\right)\right\}^{-1}$ belongs to $\mathscr{U}^{u}$ or $\mathscr{L}^{u}$ according as $c>C_{0}$ or $c<C_{0}$.

Now we consider the following condition (A):

(A.1) For suitably chosen constants $\alpha, \beta, C_{3}, C_{4}$ and $\delta$ such that $0<\alpha<2$, $-\infty<\beta<\infty, 0<C_{3}<C_{4}<\infty$ and $0<\delta<1$, it holds that for any $h \in(0, \delta)$

$$
C_{3} h^{\alpha} /|\log h|^{\beta} \leqq \sigma^{2}(h) \leqq C_{4} h^{\alpha} /|\log h|^{\beta}\left({ }^{6}\right),
$$

(A.2) $\sigma^{2}(h)$ is concave or convex in $(0, \delta)$, where $\delta$ is a constant mentioned in (A.1).

REMARK 2. The condition (A.1) is a slight generalization of the corresponding one in Beljaev's case, because $\beta$ is arbitrary in our case.

REMARK 3. For the existence of processes satisfying the condition (A), we have the following sufficient condition.

A sufficient condition: Let $f$ be the spectral density function of correlation function $\rho$. If $f$ satisfies the following two conditions, then $X$ satisfies the condition (A).

(i) There exist positive constants $C_{3}, C_{4}$ and $K$ such that

$$
C_{3} \leqq f(x) x^{\alpha+1}(\log x)^{\beta} \leqq C_{4}, \quad x \geqq K,
$$

where $0<\alpha<2,-\infty<\beta<\infty$.

(ii) $g(x)=x^{2} f(x)$ is two times differentiable in $x$, and for some $0<\varepsilon<1$ either one of the following (a) or (b) holds.

(a) $x^{3-\varepsilon} g^{\prime \prime}(x)$ is bounded from below, and $\lim _{\inf } \rightarrow \infty x^{3-\varepsilon} g^{\prime \prime}(x)>0$.

(b) $x^{3-\varepsilon} g^{\prime \prime}(x)$ is bounded from above, and lim $\sup _{x \rightarrow \infty} x^{3-\varepsilon} g^{\prime \prime}(x)<0$.

The proof of this statement will be given in $§ 7$. It is also shown that, the cases (a) and (b) correspond to the convexity and concavity of $\sigma^{2}$, respectively.

$\left.{ }^{(}\right)$If a separable stationary Gaussian process $X$ satisfy the condition (A.1), then $x(t, \omega)$ is continuous in $t$ with probability 1 (cf. [4]). 
Now let $f$ be a spectral density such that

$$
f(x)=c /\left(x^{\alpha+1}(\log x)^{\beta}\right), \quad x \geqq K,
$$

where $c>0, K \geqq 1,0<\alpha<2$ and $-\infty<\beta<\infty$. If $f^{\prime}(x)$ and $f^{\prime \prime}(x)$ are bounded on $[0, K]$, then the corresponding process $X$ satisfies the condition (A), and $\sigma^{2}(h)$ is convex or concave in a small interval $(0, \delta)$ according as $1<\alpha<2$ (or $\alpha=1, \beta>0)$ or $0<\alpha<1$ (or $\alpha=1, \beta<0$ ). In fact, we have for $g(x)=x^{2} f(x)$

$$
\frac{1}{c} g^{\prime \prime}(x)=\frac{1}{x^{\alpha+1}(\log x)^{\beta}}\left\{\alpha(\alpha-1)+\frac{(2 \alpha-1) \beta}{\log x}+\frac{\beta(\beta+1)}{(\log x)^{2}}\right\}
$$

Then we can see for $0<\varepsilon<2-\alpha$

$$
\liminf _{x \rightarrow \infty} x^{3-\varepsilon} g^{\prime \prime}(x)=\infty, \quad 1<\alpha<2 \text { or } \alpha=1 \text { and } \beta>0
$$

and

$$
\limsup _{x \rightarrow \infty} x^{3-\varepsilon} g^{\prime \prime}(x)=-\infty, \quad 0<\alpha<1 \text { or } \alpha=1, \quad \beta<0,
$$

which show the convexity and concavity of $\sigma^{2}$ respectively. In the case $\alpha=1, \beta=0$, $\sigma^{2}$ is still concave if other conditions hold. But if we replace constant $c$ by a positive and bounded function, then there may happen both cases.

Now we state:

THEOREM 2. Let $\varphi$ be a positive, continuous and nondecreasing function defined on $[a, \infty)$ with $a>0$. If the process satisfies the condition (A) and it holds that

$$
\int_{a}^{\infty} \varphi(t)^{4 / \alpha-1} \exp \left[-\frac{1}{2} \varphi^{2}(t)\right] d t<\infty
$$

then the function $\varphi$ belongs to $\mathscr{U}^{u}$.

Under the same assumption on $X$ as in Theorem 2, we have

COROLlary 2.1. For any $\varepsilon>0$,

$\left\{2 \log t+(4 / \alpha+1) \log _{(2)} t+2 \log _{(3)} t+\cdots+2 \log _{(n-1)} t+(2+\varepsilon) \log _{(n)} t\right\}^{1 / 2} \in \mathscr{U}^{u}$.

COROLlaRY 2.2 .

$P\left(\lim _{h \downarrow 0} \sup \left[\frac{x(t)-x(s)}{\sigma(t-s)\{2|\log | t-s||\}^{1 / 2}} ; 0 \leqq s, t \leqq 1,0<|t-s| \leqq h\right] \leqq 1\right)=1$.

THEOREM 3. Let $\varphi$ be a positive, continuous and nondecreasing function defined on $[a, \infty)$ with $a>0$. If the process $X$ satisfies the condition (A.1) and $\sigma^{2}$ is concave in $a$ small interval $(0, \delta)$, and further

$$
\int_{a}^{\infty} \varphi(t)^{4 / \alpha-1} \exp \left[-\frac{1}{2} \varphi^{2}(t)\right] d t=\infty
$$

then the function $\varphi$ belongs to $\mathscr{L}^{u}$. 
Under the same assumption on $X$ as in Theorem 3, it follows

COROLlaRY 3.1. For any $\varepsilon \geqq 0$,

$\left\{2 \log t+(4 / \alpha+1) \log _{(2)} t+2 \log _{(3)} t+\cdots+2 \log _{(n-1)} t+(2-\varepsilon) \log _{(n)} t\right\}^{1 / 2} \in \mathscr{L}^{u}$.

Combining Corollary 2.1 and 3.1, we have

COROLlaRY 3.2. Under the same assumption on $X$ as in Theorem 3, it holds that $P\left(\lim _{h \downarrow 0} \sup \left[\frac{x(t)-x(s)}{\sigma(t-s)\{2|\log | t-s||\}^{1 / 2}} ; 0 \leqq s, t \leqq 1,0<|t-s| \leqq h\right]=1\right)=1$.

REMARK 4. As was stated already, our main purpose is to prove Theorems 2 and 3. But we remark here that the condition (A) excludes all the cases for $\alpha=0$ which contains the critical case whether all sample functions are continuous or not (cf. X. Fernique [4]).

Next, we shall state the corresponding results concerning the local continuity of $X$.

Definition 2. Let $\psi$ be a function defined on $[a, \infty)$ with $a>0$. If, for almost all $\omega$, there exists a positive $\delta(\omega)$ such that $0<h<\delta(\omega)$ implies $|x(h, \omega)-x(0, \omega)|$ $<\sigma(h) \psi(1 / h)$, then $\psi$ is called a function belonging to the upper class with respect to the local continuity of $X$. If, for almost all $\omega$, there does not exist any positive $\delta$ with the above stated property, then $\psi$ is called a function belonging to the lower class with respect to the local continuity of $X$.

The collections of functions belonging to the upper and lower classes with respect to the local continuity of $X$ are denoted by $\mathscr{U}$ and $\mathscr{L}$, respectively.

REMARK 5. Let $t$ be a number between 0 and 1 . If $\psi$ belongs to $\mathscr{U}$, then for almost all $\omega$ there exists $\delta(t, \omega)>0$ such that

$$
|x(t+h, \omega)-x(t, \omega)|<\sigma(h) \psi(1 / h), \quad 0<h<\delta(t, \omega),
$$

because $X$ is stationary. We may also consider that the above inequality holds for any $h(\neq 0)$ between $-\delta(t, \omega)$ and $\delta(t, \omega)$, because $Y=\{y(t, \omega)=x(1-t, \omega) ; 0 \leqq t \leqq 1\}$ is stochastically equivalent to $X$.

Using the notations $\mathscr{U}$ and $\mathscr{L}$, we have

THEOREM 4. Let $\psi$ be a positive, continuous nondecreasing function on $[a, \infty)$ with $a>0, X$ be a process satisfying the condition (A.1), and $\sigma(h)$ be monotone nondecreasing for small $h>0$. If

$$
\int_{a}^{\infty} \frac{1}{t} \psi(t)^{2 / \alpha-1} \exp \left[-\frac{1}{2} \psi^{2}(t)\right] d t<\infty
$$

then $\psi$ belongs to $\mathscr{U}$.

COROLlary 4.1. Under the same assumption on $X$ as in Theorem 4,

$$
\left\{2 \log _{(2)} t+(2 / \alpha+1) \log _{(3)} t+2 \log _{(4)} t+\cdots+2 \log _{(n-1)} t+(2+\varepsilon) \log _{(n)} t\right\}^{1 / 2}
$$

belongs to $\mathscr{U}$ if $\varepsilon>0$. 
THEOREM 5. Let $\psi$ be a positive, continuous and nondecreasing function defined on $[a, \infty)$ with $a>0$. If $X$ satisfies the condition (A.1) and $\sigma^{2}$ is concave in a small interval $(0, \delta)$, and further

$$
\int_{a}^{\infty} \frac{1}{t} \psi(t)^{2 / \alpha-1} \exp \left[-\frac{1}{2} \psi^{2}(t)\right] d t=\infty
$$

then $\psi$ belongs to $\mathscr{L}$.

COROLlaRY 5.1. Under the same assumption on $X$ as in Theorem 5,

$$
\left\{2 \log _{(2)} t+(2 / \alpha+1) \log _{(3)} t+2 \log _{(4)} t+\cdots+2 \log _{(n-1)} t+(2-\varepsilon) \log _{(n)} t\right\}^{1 / 2}
$$

belongs to $\mathscr{L}$ if $\varepsilon \geqq 0$.

Combining Corollary 4.1 with the above one, we have

COROLlaRY 5.2. Under the same assumption on $X$ as in Theorem 5, it holds that

$$
P\left(\limsup _{h \downarrow 0} \frac{x(h)-x(0)}{\sigma(h)\left\{2 \log _{(2)}(1 / h)\right\}^{1 / 2}}=1\right)=1 .
$$

Let us return to the uniform continuity of $X$.

TheOREM 6. Let $0<\alpha<2,-\infty<\beta<\infty, C_{4}>0$, and suppose that

$$
\sigma^{2}(h) \leqq C_{4} h^{\alpha} /|\log h|^{\beta} .
$$

If $\sigma^{2}$ is concave or convex in a small interval $(0, \delta)$ according as $0<\alpha<1$ (or $\alpha=1$, $\beta \leqq 0)$ or $1<\alpha<2$ (or $\alpha=1, \beta>0)$, then for any $\varepsilon>0$ there exists an $h_{0}(\omega)$, with probability 1 , such that $0<|t-s|<h_{0}(\omega)$ implies

$$
|x(t, \omega)-x(s, \omega)| \leqq\left\{(2+\varepsilon) C_{4} \frac{|\dot{t}-s|^{\alpha}}{|\log | t-s||^{\beta-1}}\right\}^{1 / 2}, \quad 0 \leqq s, t \leqq 1 .
$$

The above result is an improvement of the first half of Theorem 7 in Beljaev [1]. Now let $F$ be the spectral function of $X$, i.e.

$$
\rho(h)=\int_{-\infty}^{\infty} \exp [i h x] d F(x)=2 \int_{0}^{\infty} \cos h x d F(x)\left({ }^{7}\right) .
$$

Then we have

COROLlaRY 6.1. Let $X$ be a process satisfying

$$
\int_{0}^{\infty} x^{\alpha}|\log x|^{\beta} d F(x)<\infty, \quad 0<\alpha<2, \quad-\infty<\beta<\infty .
$$

If $\sigma^{2}$ is concave or convex in a small interval $(0, \delta)$ according as $0<\alpha<1$ (or $\alpha=1$,

$\left.{ }^{7}\right) F$ is a nondecreasing function of symmetric variation with $F(\infty)-F(-\infty)=1$. 
$\beta \leqq 0)$ or $1<\alpha<2$ (or $\alpha=1, \beta>0)$, then for any $\varepsilon>0$ there exists an $h_{0}(\omega)$, with probability 1 , such that

$$
|x(t, \omega)-x(s, \omega)| \leqq \varepsilon\left\{\frac{|t-s|^{\alpha}}{|\log | t-s||^{\beta-1}}\right\}^{1 / 2}, 0 \leqq s, t \leqq 1,0<|t-s|<h_{0}(\omega) .
$$

This corresponds to Theorem 4 in G. A. Hunt [5].

3. Proof of Theorem 1. Since $\rho(t-s)$ is continuous in $(t, s) \in[0,1] \times[0,1]$, we have by Mercer's theorem

$$
\rho(t-s)=\sum_{n=1}^{\infty} \frac{1}{\lambda_{n}} \varphi_{n}(t) \varphi_{n}(s)
$$

where $\lambda_{n}, \varphi_{n}(n=1,2,3, \ldots)$ are the eigenvalues and orthonormal eigenfunctions of the integral equation

$$
\varphi(t)=\lambda \int_{0}^{1} \rho(t-s) \varphi(s) d s,
$$

and $\lambda_{n}>0$ because $\rho$ is positive definite. We remark that

$$
\begin{aligned}
\left|\varphi_{n}(t+h)-\varphi_{n}(t)\right| & =\left|\lambda_{n} \int_{0}^{1}\{\rho(t+h-s)-\rho(t-s)\} \varphi_{n}(s) d s\right| \\
& \leqq \lambda_{n}\left\{\int_{0}^{1}|\rho(t+h-s)-\rho(t-s)|^{2} d s\right\}^{1 / 2} \\
& =\lambda_{n}\left\{\int_{0}^{1}|E[(x(t+h)-x(t)) x(s)]|^{2} d s\right\}^{1 / 2} \\
& \leqq \lambda_{n} \sigma(h) .
\end{aligned}
$$

Now let $\left\{y_{n} ; n=1,2,3, \ldots\right\}$ be a sequence of mutually independent standard Gaussian random variables. Then, for any $t \in[0,1]$, the series

$$
y(t, \omega)=\sum_{n=1}^{\infty} \frac{1}{\sqrt{ } \lambda_{n}} y_{n}(\omega) \varphi_{n}(t)
$$

converges in the square mean (cf. M. Loève [6, p. 478]), and by the three series theorem, converges with probability 1 . So the Gaussian system $Y=\{y(r) ; 0 \leqq r \leqq 1\}$ where $r$ denotes rational number is equivalent to the system $X^{\prime}=\{x(r) ; 0 \leqq r \leqq 1\}$. Next we consider the events $\mathscr{E}^{\prime}(a)$ and $\mathscr{E}^{\prime \prime}(a ; j)$ defined by

$$
\begin{array}{r}
\mathscr{E}^{\prime}(a)=\left\{\omega ; \limsup _{h \downarrow 0}\left[\frac{x(r)-x\left(r^{\prime}\right)}{g\left(\left|r-r^{\prime}\right|\right)} ; 0 \leqq r, r^{\prime} \leqq 1,0<\left|r-r^{\prime}\right| \leqq h\right] \leqq a\right\}, \\
\mathscr{E}^{\prime \prime}(a ; j)=\left\{\omega ; \limsup _{h \downarrow 0}\left[\sum_{n=j}^{\infty} \frac{1}{\sqrt{ } \lambda_{n}} \frac{\varphi_{n}(r)-\varphi_{n}\left(r^{\prime}\right)}{g\left(\left|r-r^{\prime}\right|\right)} y_{n} ; 0 \leqq r, r^{\prime} \leqq 1,\right.\right. \\
\left.\left.0<\left|r-r^{\prime}\right| \leqq h\right] \leqq a\right\}, \\
j=1,2,3, \ldots,
\end{array}
$$


where $r$ and $r^{\prime}$ denote rational numbers. Since $X$ and $g$ are continuous, the equivalence of $X^{\prime}$ and $Y$ implies

$$
P(\mathscr{E}(a))=P\left(\mathscr{E}^{\prime}(a)\right)=P\left(\mathscr{E}^{\prime \prime}(a ; 1)\right) .
$$

Moreover (7) and the assumption on $\sigma(h) / g(h)$ tells

$$
\mathscr{E}^{\prime \prime}(a ; 1)=\mathscr{E}^{\prime \prime}(a ; j), \quad j=1,2,3, \ldots
$$

Therefore, using the fact that $\mathscr{E}^{\prime \prime}(a ; j)$ is a tail event, i.e. $\mathscr{E}^{\prime \prime}(a ; j)$ depends only on $y_{n}$ with $n \geqq j$, we can see by Kolmogorov's 0 -1 law $P(\mathscr{E} "(a ; 1))=0$ or 1 , and accordingly $P(\mathscr{E}(a))=0$ or 1 . Q.E.D.

4. Proof of Theorem 2. We proceed along the line of T. Sirao [7], but the proof is somewhat complicated. So we divide it into several lemmas.

LEMMA 1. Theorems 2 and 3 are true if they hold under the assumption that for $t>e^{e} \vee a\left(^{8}\right)$

$$
\{2 \log t\}^{1 / 2} \leqq \varphi(t) \leqq\left\{2 \log t+(7 / \alpha) \log _{(2)} t\right\}^{1 / 2}\left({ }^{9}\right) .
$$

Proof. Cf. T. Sirao [7, Lemma 1]. (The proof given there does not need any change for the present case.)

By Lemma 1, we may assume in the following proofs of Theorems 2 and 3 that (8) holds for any $t \geqq a$ and accordingly $\varphi(t)$ tends to infinity with $t$. Moreover Theorems 2 and 3 treat the Hölder continuity of stationary processes. So it is enough to consider the behavior of $x(t)$ in the time interval $[0, \delta]$. (Divide [0,1] into $2([1 / \delta]+1)\left({ }^{10}\right)$ subintervals of $[k \delta,(k+1) \delta],[(k+1 / 2) \delta,(k+3 / 2) \delta], k=0,1,2, \ldots$, $[1 / \delta]+1$, and consider a pair of $(t, s)$ such that $|t-s|<\delta / 2$, if necessary.) Therefore, despite the unnaturalness of assuming (A.1) in $(0,1)$, we may assume that the condition (A), especially (A.2), holds in $(0,1)$ if we regard $[0, \delta]$ as $[0,1]$ for the convenience of description. Further we may regard by the same reason that $\sigma^{2}$ is monotone nondecreasing in $(0,1)$.

Now we define the event $E(p ; k, l)$ by

$$
\begin{aligned}
& E(p ; k, l)=\left\{\omega ; x\left((k+l) / 2^{p}, \omega\right)-x\left(k / 2^{p}, \omega\right)\right.\left.\geqq \sigma\left(l / 2^{p}\right) \varphi\left(2^{p} / l\right)\right\}, \\
& 0 \leqq k \leqq 2^{p}, \quad 0 \leqq l \leqq p^{1 / \alpha}, \quad p=1,2,3, \ldots
\end{aligned}
$$

Then we have

LeMma 2. For any $c \in(0,1)$, we have

$$
\sum_{p=1}^{\infty} \sum_{k=1}^{\infty} \sum_{l=\left[c p^{1 / \alpha}\right]+1}^{\left[p^{1 / \alpha}\right]} P(E(p ; k, l))<\infty \quad \text { or }=\infty
$$

$\left(^{8}\right) a \vee b=\max (a, b)$ and $a \wedge b=\min (a, b)$.

( $\left.{ }^{9}\right)$ The coefficient 7 on the second term has no special meaning except that the integral (2) for right hand side is finite.

${ }^{(10)}[x]$ denotes the greatest integer which does not exceed $x$. 
according as

$$
\int_{a}^{\infty} \varphi(t)^{4 / \alpha-1} \exp \left[-\frac{1}{2} \varphi^{2}(t)\right] d t<\infty \quad \text { or }=\infty .
$$

Proof. By the monotonicity of $\varphi$, we may assume without loss of generality that $a$ is sufficiently large so that $\varphi(t)>1$ and accordingly $\varphi(t) \exp \left[-\varphi^{2}(t) / 2\right]$ is nonincreasing in $t \geqq a$. Then for $(p, l)$ with $2^{p} / l \geqq a$, we have

$$
\begin{aligned}
& \frac{1}{2(2 \pi)^{1 / 2}} \frac{1}{\varphi\left(2^{p} / l\right)} \exp \left[-\frac{1}{2} \varphi^{2}\left(2^{p} / l\right)\right] \\
&<P(E(p ; k, l))=\frac{1}{(2 \pi)^{1 / 2}} \int_{\varphi\left(2^{p} / l\right)}^{\infty} \exp \left[-x^{2} / 2\right] d x \\
&<\frac{1}{(2 \pi)^{1 / 2}} \frac{1}{\varphi\left(2^{p} / l\right)} \exp \left[-\frac{1}{2} \varphi^{2}\left(2^{p} / l\right)\right] .
\end{aligned}
$$

We can further see by the monotonicity of $\varphi$ that

$$
\begin{aligned}
\frac{1}{\varphi\left((1 / c) p^{-1 / \alpha} 2^{p}\right)} \exp \left[-\frac{1}{2} \varphi^{2}\left(\frac{1}{c} p^{-1 / \alpha} 2^{p}\right)\right] \\
\leqq \frac{1}{\varphi\left(2^{p} / l\right)} \exp \left[-\frac{1}{2} \varphi^{2}\left(2^{p} / l\right)\right] \\
\leqq \frac{1}{\varphi\left(p^{-1 / \alpha} 2^{p}\right)} \exp \left[-\frac{1}{2} \varphi^{2}\left(p^{-1 / \alpha} 2^{p}\right)\right], \quad\left[c p^{1 / \alpha}\right] \leqq l \leqq\left[p^{1 / \alpha}\right] .
\end{aligned}
$$

So if $p_{0}$ and $C_{5}$ are so large that

$$
\varphi\left(p_{0}^{-1 / \alpha} 2^{p_{0}}\right) \wedge\left(p_{0}^{-1 / \alpha} 2^{p_{0}}\right)>a \text { and } C_{5} \geqq \frac{1}{(2 \pi)^{1 / 2}}\left\{1-\frac{1}{2\left(1-p_{0}^{-1}\right)^{1 / \alpha}}\right\}^{-1},
$$

then it follows from (10), (11) and (8) that

$$
\begin{aligned}
\sum_{p=p_{0}}^{\infty} \sum_{k=1}^{2^{p}} \sum_{l=\left[c p^{1 / \alpha}\right]+1}^{\left[p^{1 / \alpha}\right]} P(E(p ; k, l)) \\
\quad \leqq \frac{1}{(2 \pi)^{1 / 2}} \sum_{p=p_{0}}^{\infty} \frac{2^{p} p^{1 / \alpha}}{\varphi\left(p^{-1 / \alpha} 2^{p}\right)} \exp \left[-\frac{1}{2} \varphi^{2}\left(p^{-1 / \alpha} 2^{p}\right)\right] \\
\quad \leqq C_{5} \sum_{p=p_{0}}^{\infty}\left\{\frac{2^{p}}{p^{1 / \alpha}}-\frac{2^{p-1}}{(p-1)^{1 / \alpha}}\right\} p^{2 / \alpha} \frac{1}{\varphi\left(p^{-1 / \alpha} 2^{p}\right)} \exp \left[-\frac{1}{2} \varphi^{2}\left(p^{-1 / \alpha} 2^{p}\right)\right] \\
\leqq C_{5} \int_{a}^{\infty} \varphi(t)^{4 / \alpha-1} \exp \left[-\frac{1}{2} \varphi^{2}(t)\right] d t .
\end{aligned}
$$

Similarly we can see the existence of $C_{6}$ such that

$$
\begin{aligned}
& \sum_{p=p_{0}}^{\infty} \sum_{k=1}^{2^{p}} \sum_{l=\left[c p^{1 / \alpha}\right]+1}^{[p 1 / \alpha]} P(E(p ; k, l)) \\
& \geqq \frac{(1-c)}{2(2 \pi)^{1 / 2}} \sum_{p=p_{0}}^{\infty} \frac{2^{p} p^{1 / \alpha}}{\varphi\left(c^{-1} p^{-1 / \alpha} 2^{p}\right)} \exp \left[-\frac{1}{2} \varphi^{2}\left(c^{-1} p^{-1 / \alpha} 2^{p}\right)\right] \\
& \quad \geqq C_{6} \sum_{p=p_{0}}^{\infty}\left(\frac{2^{p+1}}{c(p+1)^{1 / \alpha}}-\frac{2^{p}}{c p^{1 / \alpha}}\right) \varphi\left(c^{-1} p^{-1 / \alpha} 2^{p}\right)^{4 / \alpha-1} \exp \left[-\frac{1}{2} \varphi^{2}\left(c^{-1} p^{-1 / \alpha} 2^{p}\right)\right] \\
& \quad \geqq C_{6} \int_{c-1 p_{0}^{-1 / \alpha} 2_{0}}^{\infty} \varphi(t)^{4 / \alpha-1} \exp \left[-\frac{1}{2} \varphi^{2}(t)\right] d t
\end{aligned}
$$


Now (12) and (13) prove Lemma 2. Q.E.D.

According to Lemma 2, we can see in the present case

$$
\sum_{p=1}^{\infty} \sum_{k=1}^{2^{p}} \sum_{l=\left[p^{1 / \alpha} / 2\right]}^{\left[p^{1 / \alpha}\right]} P(E(p ; k, l))<\infty .
$$

This means by Borel-Cantelli's lemma that for almost all $\omega$ there exists $p_{0}(\omega)$ such that $p>p_{0}(\omega)$ implies

$$
x\left((k+l) / 2^{p}, \omega\right)-x\left(k / 2^{p}, \omega\right)<\sigma\left(l / 2^{p}\right) \varphi\left(2^{p} / l\right), \quad 0 \leqq k \leqq 2^{p},\left[\frac{1}{2} p^{1 / \alpha}\right] \leqq l \leqq\left[p^{1 / \alpha}\right] .
$$

It is our purpose to show that the above inequality holds for any pair $(s, t)$ with $0<|t-s|<1 / 2^{p_{0}(\omega)}$. To show this, we consider the following events $F(q ; m, n)$ for fixed $(p, k, l)$. Let $c$ be a large number which makes $e^{c}$ an integer and satisfies inequalities

$$
\begin{aligned}
(2 / \pi)^{1 / 2} \exp \left[-\frac{C_{7}^{2}}{8}\left(e^{c / 4} / 2\right)^{2 \alpha(q-1)}\right] & <C_{7} e^{-3 q c}, \quad q \geqq 2, \\
C_{7}\left(e^{c / 4} / 2\right)^{\alpha(q-1)} & >2 C_{8}
\end{aligned}
$$

where $C_{7}$ and $C_{8}$ are positive constants to be defined later. Now let us set $b_{1}=k / 2^{p}$, $b_{2}=(k+l) / 2^{p}, h_{q}=\exp [-q c] / 2^{p}$ and

$$
\begin{array}{r}
F(q ; m, n)=\left\{\omega ; x\left(b_{2}-m h_{q}, \omega\right)-x\left(b_{1}+n h_{q}, \omega\right)\right. \\
\left.\geqq \sigma\left(b_{2}-b_{1}-(m+n) h_{q}\right)\left\{\varphi+\frac{2 c}{\varphi} \sum_{i=0}^{q-1} \frac{1}{2^{\alpha i}}\right\}\right\}, \\
0 \leqq m, n \leqq \exp [q c],
\end{array}
$$

where $\varphi$ denotes $\varphi\left(2^{p} / l\right)=\varphi\left(1 /\left(b_{2}-b_{1}\right)\right)$. Further set

$$
S_{q}=\{m ; 0 \leqq m \leqq \exp [q c]\}, \quad F_{q}=\bigcup_{m, n \in S_{q}} F(q ; m, n),
$$

and denote the smallest integer $p^{\prime}$ satisfying (16) by $p_{1}$

$$
2^{p+1} \geqq p^{1 / \alpha}, \quad p \log 2 \geqq 2 \log p / \alpha .
$$

The following lemma plays an essential role, and we need several lemmas to prove it.

LEMMA 3. Under the assumption of Theorem 2, there exists an absolute constant $\left({ }^{11}\right)$ $C_{9}$ such that for $p \geqq p_{1}$

$$
P\left(F_{q}\right) \leqq C_{9} P(E(p ; k, l)), \quad q \geqq 1 .
$$

Proof. We consider a fixed triple $(p, k, l)$ with relation of $p \geqq p_{1}$. Denoting the

(11) An absolute constant means a constant independent of $(p, k, l)$ and $q$. 
complementary event of $F$ by $F^{\prime}$, we have

$$
\begin{aligned}
P\left(F_{q}\right) & =P\left(F_{q-1}\right)+P\left(F_{q-1}^{\prime} \cap F_{q}\right) \\
& \leqq P\left(F_{q-1}\right)+\sum_{m, n \in S_{q}} P\left(F_{q-1}^{\prime} \cap F(q ; m, n)\right), \quad q \geqq 2 .
\end{aligned}
$$

Let us denote the second term in the right-hand side of (18) by $P_{1}$ and estimate it. We denote the interval $\left(b_{1}+n h_{q}, b_{2}-m h_{q}\right)$ by $I(q ; m, n)$. Next, for each pair $(m, n)$ of $m, n \in S_{q}$, we choose two elements $m_{1}, n_{1}$ of $S_{q-1}$ in such a way that

$$
\left|m h_{q}-m_{1} h_{q-1}\right|, \quad\left|n h_{q}-n_{1} h_{q-1}\right| \leqq h_{q-1},
$$

and, if $\rho$ is convex in $(0, \delta), I(q ; m, n) \subset I\left(q-1 ; m_{1}, n_{1}\right)$ and, if $\rho$ is concave in $(0, \delta)$,

$$
I(q ; m, n)-I\left(q-1 ; m_{1}, n_{1}\right) \neq \varnothing, \quad I\left(q-1 ; m_{1}, n_{1}\right)-I(q ; m, n) \neq \varnothing .
$$

Then we have

$$
\begin{aligned}
P_{1} & =\sum_{m, n \in S_{q}} P\left(F_{q-1}^{\prime} \cap F(q ; m, n)\right) \\
& \leqq \sum_{m, n \in S_{q}} P\left(F\left(q-1 ; m_{1}, n_{1}\right)^{\prime} \cap F(q ; m, n)\right) .
\end{aligned}
$$

To estimate the right-hand side of (20), we use the following lemmas.

LEMMA 4. (i) If the correlation function $\rho$ is convex in $(0,1)$, then it holds that for any pair of nonoverlapping intervals $(a, b),(c, d) \subset(0,1)$

$$
E((x(b)-x(a))(x(d)-x(c))) \leqq 0,
$$

and for $(c, d) \subset(a, b) \subset(0,1)$

$$
\sigma^{2}(d-a)+\sigma^{2}(b-c) \geqq \sigma^{2}(b-a)+\sigma^{2}(d-c) .
$$

(ii) If the correlation function $\rho$ is concave in $(0,1)$, then it holds that for any pair of nonoverlapping intervals $(a, b),(c, d) \subset(0,1)$

$$
E((x(b)-x(a))(x(d)-x(c))) \geqq 0,
$$

and for any pair of overlapping intervals $(a, b),(c, d) \subset(0,1)$

$$
\sigma^{2}(d-a)+\sigma^{2}(b-c) \geqq \sigma^{2}(b-a)+\sigma^{2}(d-c) .
$$

Proof. We prove only (i) because (ii) can be proved similarly. Let $0 \leqq a \leqq b \leqq c$ $\leqq d \leqq 1$. Then we have

$$
E((x(b)-x(a))(x(d)-x(c)))=\{\rho(d-b)-\rho(d-a)\}-\{\rho(c-b)-\rho(c-a)\} .
$$

Since $(d-b)-(d-a)=(c-b)-(c-a)$ and $\rho$ is convex in $(0,1)$, the right-hand side of the above equality is nonpositive. So we have (21). 
Next, let $0 \leqq a \leqq c \leqq d \leqq b \leqq 1$. Then we have

$$
\begin{aligned}
\left\{\sigma^{2}(d-a)+\sigma^{2}(b-c)\right\}-\left\{\sigma^{2}(b-a)\right. & \left.+\sigma^{2}(d-c)\right\} \\
& =2\{\rho(b-a)+\rho(d-c)\}-2\{\rho(d-a)+\rho(b-c)\} \\
& \geqq 0,
\end{aligned}
$$

because $d-c \leqq(d-a) \wedge(b-c) \leqq b-a$ and $(b-a)+(d-c)=(d-a)+(b-c)$. Q.E.D

LEMMA 5. Let $(U, V)$ be a two dimensional Gaussian random variable with $E(U)=E(V)=0$ and $E\left(U^{2}\right)=E\left(V^{2}\right)=1$. Then, for a pair $(a, b)$ with $0<a<b$, the function $p(a, b ; \rho)=P(U<a, V>b)$ is monotone decreasing in $\rho=E(U V)$.

Proof. See Lemma 2 in T. Sirao [7].

Let us set, for any $m, n \in S_{q}$ and $m_{1}, n_{1} \in S_{q-1}$ which are chosen by the way stated already, $a=b_{1}+n h_{q}, b=b_{2}-m h_{q}, c=b_{1}+n_{1} h_{q-1}$ and $d=b_{2}-m_{1} h_{q-1}$.

LEMMA 6. Let $\rho$ be the correlation coefficient between $x(b)-x(a)$ and $x(d)-x(c)$. Then there exists a positive constant $C_{10}$ such that

$$
\rho \geqq 1-C_{10}(1 / p) \exp [-(q-1) c \alpha / 2] \text {. }
$$

Proof. We can see from (22) and (24) that

$$
\sigma^{2}(d-a)+\sigma^{2}(b-c) \geqq \sigma^{2}(b-a)+\sigma^{2}(d-c) .
$$

Hence we have

$$
\begin{aligned}
E((x(b)-x(a))(x(d)-x(c))) & =\frac{1}{2}\left\{\sigma^{2}(d-a)+\sigma^{2}(b-c)-\sigma^{2}(d-b)-\sigma^{2}(c-a)\right\} \\
& \geqq \frac{1}{2}\left\{\sigma^{2}(b-a)+\sigma^{2}(d-c)-\sigma^{2}(d-b)-\sigma^{2}(c-a)\right\} .
\end{aligned}
$$

So it follows from the monotonicity of $\sigma^{2}$ that

Now put

$$
\begin{aligned}
\rho & \geqq 1-\frac{1}{2} \frac{\sigma^{2}(d-b)+\sigma^{2}(c-a)}{\sigma(b-a) \sigma(d-c)} \\
& \geqq 1-\frac{\sigma^{2}\left(h_{q+1}\right)}{\sigma^{2}\left(b_{2}-b_{1}-2 h_{q} \exp [q c]\right)} .
\end{aligned}
$$

$$
\begin{array}{r}
M=\sup \left\{\frac{p}{(l-2)^{\alpha} \exp [(q-1) c \alpha / 2]}\left|\frac{p \log 2-\log (l-2)}{p \log 2+(q-1) c}\right|^{\beta} ; p \geqq p_{1}, q \geqq 2,\right. \\
\left.\left[\frac{1}{2} p^{1 / \alpha}\right] \leqq l \leqq\left[p^{1 / \alpha}\right], c \geqq 2\right\} .
\end{array}
$$

Evidently, by (16), $M$ is finite. Then we have by (A.1)

$$
\begin{aligned}
\rho & \geqq 1-\frac{C_{4}}{C_{3}}\left\{\frac{l}{(l-2) \exp [(q-1) c]}\right\}^{\alpha}\left|\frac{p \log 2-\log (l-2)}{p \log 2+(q-1) c}\right|^{\beta} \\
& \geqq 1-\frac{C_{4}}{C_{3}} \frac{M}{p} \exp [-(q-1) c \alpha / 2] .
\end{aligned}
$$

So, putting $C_{10}=C_{4} M / C_{3}$, we have (25). Q.E.D. 
Now let us go back to the estimation of $P_{1}$. Let $Y$ and $Z$ be mutually independent Gaussian random variables with $E(Y)=E(Z)=0$ and $E\left(Y^{2}\right)=E\left(Z^{2}\right)=1$. Then, using the notations in Lemma 6, we have

$$
\begin{aligned}
& P\left(F \left(q-1 ; m_{1},\right.\right.\left.\left.n_{1}\right)^{\prime} \cap F(q ; m, n)\right) \\
&= P\left(x(b)-x(a) \geqq \sigma(b-a)\left(\varphi+\frac{2 c}{\varphi} \sum_{i=0}^{q-1} 2^{-\alpha i}\right),\right. \\
&\left.x(d)-x(c)<\sigma(d-c)\left(\varphi+\frac{2 c}{\varphi} \sum_{i=0}^{q-2} 2^{-\alpha i}\right)\right) \\
&=P\left(Y \geqq \varphi+\frac{2 c}{\varphi} \sum_{i=0}^{q-1} 2^{-\alpha i},\left(1-\rho^{2}\right)^{1 / 2} Z+\rho Y<\varphi+\frac{2 c}{\varphi} \sum_{i=0}^{q-2} 2^{-\alpha i}\right) .
\end{aligned}
$$

Put $\rho_{0}=1-C_{10} \exp [-(q-1) c \alpha / 2] / p$, and we can see from (25), (26) and Lemma 5 that

$$
\begin{aligned}
& P\left(F\left(q-1 ; m_{1}, n_{1}\right)^{\prime} \cap F(q ; m, n)\right) \\
& \leqq P\left(Y \geqq \varphi+\frac{2 c}{\varphi} \sum_{i=0}^{q-1} 2^{-\alpha i},\left(1-\rho_{0}^{2}\right)^{1 / 2} Z<\left(1-\rho_{0}\right)\left(\varphi+\frac{2 c}{\varphi} \sum_{i=0}^{q-2} 2^{-\alpha i}\right)\right.\left.-\rho_{0} \frac{2 c}{\varphi} 2^{-\alpha(q-1)}\right) \\
&=P\left(Y \geqq \varphi+\frac{2 c}{\varphi} \sum_{i=0}^{q-1} 2^{-\alpha i}\right) P\left(Z<\left(1-\rho_{0}^{2}\right)^{-1 / 2}\right.\left.\cdot\left\{\left(1-\rho_{0}\right)\left(\varphi+\frac{2 c}{\varphi} \sum_{i=0}^{q-2} 2^{-\alpha i}-\rho_{0} \frac{2 c}{\varphi} 2^{-\alpha(q-1)}\right)\right\}\right) \\
&=P_{2} \text { (say). }
\end{aligned}
$$

Now we have by Lemma 1

$$
\varphi=\varphi\left(2^{p} / l\right) \leqq\{2 p \log 2+7 \log \log p / \alpha\}^{1 / 2} .
$$

Hence there exist absolute constants $c, C_{8}$ and $C_{7}$ such that they satisfy (15) and the following two inequalities

$$
\begin{gathered}
\left(1-\rho_{0}^{2}\right)^{-1 / 2}\left(1-\rho_{0}\right)\left(\varphi+\frac{2 c}{\varphi} \sum_{i=0}^{q-2} 2^{-\alpha i}\right) \leqq C_{8}<\infty, \\
\left(1-\rho_{0}^{2}\right)^{-1 / 2} \rho_{0} \frac{2 c}{\varphi} \geqq C_{7} \exp \left[\frac{1}{4} \alpha(q-1) c\right] .
\end{gathered}
$$

So we can see by (15) and (10)

$$
\begin{aligned}
P_{2} & \leqq P(Y \geqq \varphi) P\left(Z<C_{8}-C_{7}\left(e^{c / 4} / 2\right)^{\alpha(q-1)}\right) \\
& \leqq P(Y \geqq \varphi) P\left(Z>\frac{C_{7}}{2}\left(e^{c / 4} / 2\right)^{\alpha(q-1)}\right) \\
& \leqq P(Y \geqq \varphi)\left(\frac{1}{2 \pi}\right)^{1 / 2} \frac{2}{C_{7}}\left(e^{c / 4} / 2\right)^{-\alpha(q-1)} \exp \left[-\frac{C_{7}^{2}}{8}\left(e^{c / 4} / 2\right)^{2 \alpha(q-1)}\right] \\
& <e^{-3 q c} P(E(p ; k, l)) .
\end{aligned}
$$


Then it follows from (27) that

$$
P\left(F\left(q-1 ; m_{1}, n_{1}\right)^{\prime} \cap F(q, m, n)\right) \leqq e^{-3 q c} P(E(p ; k, l)), \quad m, n \in S_{q}, q \geqq 2,
$$

and accordingly

$$
P_{1} \leqq e^{-q c} P(E(p ; k, l))
$$

Since

$$
P\left(F_{1}\right) \leqq \sum_{m, n \in S_{1}} P(F(1 ; m, n))=e^{2 c} P(E(p ; k, l)),
$$

we get from (18) and (28)

$$
P\left(F_{q}\right) \leqq\left(e^{2 c}+\sum_{i=2}^{\infty} e^{-i c}\right) P(E(p ; k, l)), \quad q \geqq 2,
$$

which proves Lemma 3. Q.E.D.

Now we are in a position to prove Theorem 2. Set

$$
G(p ; k, l)=\bigcup_{n=1}^{\infty} \bigcap_{q=n}^{\infty} F_{q}
$$

and

$$
H(p ; k, l)=\left\{\omega ; \sup \frac{x(t)-x(s)}{\sigma(t-s)} \geqq \varphi\left(2^{p} / l\right)+\frac{2 c}{\varphi\left(2^{p} / l\right)} \sum_{i=0}^{\infty} 2^{-\alpha i}\right\},
$$

where $s$ and $t$ runs over the intervals $\left[k 2^{-p},(k+1) 2^{-p}\right]$ and $\left[(k+l-1) 2^{-p},(k+l) 2^{-p}\right]$ respectively. Then it follows from the continuity of $X$ and Lemma 3 that

$$
\begin{aligned}
P(H(p ; k, l)) & \leqq P(G(p ; k, l)) \\
& \leqq \liminf _{q \rightarrow \infty} P\left(F_{q}\right) \\
& \leqq C_{9} P(E(p ; k, l)) .
\end{aligned}
$$

Therefore we have by Lemma 2

$$
\sum_{p=p_{1}}^{\infty} \sum_{k=0}^{2^{p}} \sum_{l=\left[p^{1 / \alpha} / 2\right]+1}^{\left[p p^{1 / \alpha}\right]} P(H(p ; k, l))<\infty .
$$

According to Borel-Cantelli's lemma, (29) shows that for almost all $\omega$ there exists $p_{2}(\omega)$ such that

$$
\omega \notin H(p ; k, l) \text { for } p \geqq p_{2}(\omega), \quad 0 \leqq k \leqq 2^{p}, \quad\left[\frac{1}{2} p^{1 / \alpha}\right]<l \leqq\left[p^{1 / \alpha}\right] .
$$

Now, for any pair $(s, t)$ satisfying $0 \leqq s<t \leqq 1$ and $0<t-s<p_{2}(\omega)^{1 / \alpha} / 2^{-p_{2}(\omega)}$, choose $p, k$ and $l$ such that

$$
\frac{(p+1)^{1 / \alpha}}{2^{p+1}} \leqq t-s<\frac{p^{1 / \alpha}}{2^{p}}, \quad \frac{k}{2^{p}} \leqq s<\frac{k+1}{2^{p}}<\frac{k+l-1}{2^{p}} \leqq t<\frac{k+l}{2^{p}} .
$$


Then it holds that $p_{2}(\omega) \leqq p, 0 \leqq k<2^{p}$ and $p^{1 / \alpha} / 2 \leqq l \leqq p^{1 / \alpha}$. Accordingly the fact that $\omega \notin H(p ; k, l)$ implies

$$
\begin{aligned}
x(t, \omega)-x(s, \omega) & <\sigma(t-s)\left\{\varphi\left(2^{p} / l\right)+\frac{2 c}{\varphi\left(2^{p} / l\right)} \sum_{i=0}^{\infty} 2^{-\alpha i}\right\} \\
& \leqq \sigma(t-s)\left\{\varphi(1 /(t-s))+\frac{2 c}{\varphi(1 /(t-s))} \sum_{i=0}^{\infty} 2^{-\alpha i}\right\} .
\end{aligned}
$$

Then, taking into consideration the symmetry of a Gaussian process, we can see that $\varphi+2 c^{\prime} / \varphi$ belongs to $\mathscr{U}^{u}$, where $c^{\prime}=c \sum_{i=0}^{\infty} 1 / 2^{\alpha i}$. Since this result is obtained from the assumption of convergence of (2), the same result also should hold for $\tilde{\varphi}(t)=\varphi(t)-3 c^{\prime} / \varphi(t)$, because $\tilde{\varphi}$ is nondecreasing, continuous on $[a, \infty)$ and further the integral (2) for $\tilde{\varphi}$ is finite. Moreover $\tilde{\varphi}(t)+2 c^{\prime} \mid \tilde{\varphi}(t)<\varphi(t)$ for large $t$, as is easily seen. Hence $\varphi$ should belong to $\mathscr{U}^{u}$. Q.E.D.

5. Proof of Theorem 3. We shall use the same notations as in $\S 4$.

According to Lemma 2, the divergence of integral (3) implies

$$
\sum_{p=1}^{\infty} \sum_{k=0}^{2^{p}} \sum_{l=\left[p^{1 / \alpha} / 3\right]}^{[p 1 / \alpha]} P(E(p ; k, l))=\infty .
$$

By the definition of $\mathscr{L}^{u}$, the function $\varphi$ belongs to $\mathscr{L}^{u}$ if $E(p ; k, l)$ occurs "infinitely often" for almost all $\omega$. To prove this is the case, we apply the Chung-Erdös lemma $\left({ }^{12}\right)$.

In the sequel, we often denote $E(p ; k, l)$ by $E_{n}$, where the subscript $n$ is given in the following way. Let $E_{n}=E(p ; k, l)$ and $E_{m}=E\left(p^{\prime} ; k^{\prime}, l^{\prime}\right)$. Then $n$ stands before $m$ if and only if either one of the following hold: (i) $p<p^{\prime}$, (ii) $p=p^{\prime}$ and $l^{\prime}<l$, (iii) $p=p^{\prime}, l=l^{\prime}$ and $k<k^{\prime}$. Hence $n<m$ implies $l^{\prime} / 2^{p^{\prime}} \leqq l / 2^{p}$, where $(p, l)$ and $\left(p^{\prime}, l^{\prime}\right)$ correspond to $E_{n}$ and $E_{m}$ respectively.

Now, by the Chung-Erdös lemma and (30), it suffices to prove the following Lemma 7 and Lemma 8.

LEMMA 7. For every pair of $(n, h)$ with $n \geqq h$, there exist $c(h)>0$ and $H(n, h)>n$ such that for any $m \geqq H(n, h)$

$$
P\left(E_{m} / E_{h}^{\prime} \cap E_{h+1}^{\prime} \cap \cdots \cap E_{n}^{\prime}\right) \geqq c(h) P\left(E_{m}\right)\left({ }^{13}\right) .
$$

LEMMA 8. There exist two absolute constants $K_{1}$ and $K_{2}$ with the following property: to each $E_{j}$ there corresponds a set of events $\left\{E_{j_{i}} ; i=1,2,3, \ldots, s\right\} \subset$ $\left\{E_{n} ; n=1,2,3, \ldots\right\}$ such that

$$
\sum_{i=1}^{s} P\left(E_{j} \cap E_{f_{i}}\right) \leqq K_{1} P\left(E_{j}\right)
$$

$\left({ }^{12}\right)$ See [2].

$\left.{ }^{(13}\right) P(E / F)$ denotes the conditional probability of $E$ under the condition $F$. 
and that for all $E_{k} \neq E_{j_{i}}(i=1,2,3, \ldots, s)$ provided $k>j$

$$
P\left(E_{j} \cap E_{k}\right) \leqq K_{2} P\left(E_{j}\right) P\left(E_{k}\right) .
$$

Before we prove Lemma 7, we shall state a remark and Lemma 9. For each $E_{n}=E(p ; k, l)$, we define $U_{n}=U(p ; k, l)$ by

$$
U_{n}=U(p ; k, l)=x\left((k+l) / 2^{p}\right)-x\left(k / 2^{p}\right),
$$

where $U(p ; k, l)$ is used when we want to emphasize that $U_{n}$ corresponds to $E(p ; k, l)$. Then we have for every $n$

$$
\lim _{m \rightarrow \infty} \rho\left(U_{n}, U_{m}\right)=0,
$$

where $\rho(U, V)$ denotes the correlation coefficient between $U$ and $V$. In fact, (34) is proved in the following way. Let $U_{n}=x(b)-x(a)$ and $U_{m}=x(d)-x(c)$. Since we may regard $\sigma^{2}$ as a monotone and concave function as was remarked already, it holds by (21)

$$
\begin{aligned}
\rho\left(U_{n}, U_{m}\right) & \leqq \frac{\sigma^{2}(d-c)}{\sigma(b-a) \sigma(d-c)} \\
& =\frac{\sigma(d-c)}{\sigma(b-a)} \rightarrow 0 \quad \text { as } m \rightarrow \infty,
\end{aligned}
$$

because $d-c$ tends to 0 as $m \rightarrow \infty$.

LEMMA 9. Let $\left\{X_{1}, X_{2}, \ldots, X_{k}, Y_{m} ; m=1,2,3, \ldots\right\}$ be a sequence of standard Gaussian random variables, and assume that $\rho_{m}=\max \left\{\left|\rho_{i, m}\right| ; 0 \leqq i \leqq k\right\} \rightarrow 0$ as $m \rightarrow \infty$, where $\rho_{i, m}$ denotes $E\left(X_{i} Y_{m}\right)$. Then, for any sequence of Borel sets $B_{m} \subset$ $\left[\rho_{m}^{-c}, \rho_{m}^{c}\right]$ provided $0 \leqq c<1$ and for any bounded Borel sets $A_{i}(i=1,2, \ldots, k)$, it holds

$$
P\left(Y_{m} \in B_{m} / X_{i} \in A_{i}, i=1,2, \ldots, k\right) / P\left(Y_{m} \in B_{m}\right) \rightarrow 1 \text { as } m \rightarrow \infty .
$$

Proof. See Lemma 4 in T. Sirao [7].

Now we shall prove Lemma 7.

Proof of Lemma 7. Let us set for $U_{n}=U(p ; k, l)$ and $c>0$

$$
\begin{aligned}
& F_{n}(c)=\left\{\omega ; \varphi\left(2^{p} / l\right) \leqq U_{n} / \sigma\left(l / 2^{p}\right) \leqq \varphi\left(2^{p} / l\right)+c\right\}, \\
& E_{n}(c)=\left\{\omega ; U_{n}+c \geqq 0\right\} .
\end{aligned}
$$

Then we have by (10)

$$
\begin{aligned}
P\left(F_{n}(c)\right) & \geqq \frac{1}{2(2 \pi)^{1 / 2}} \frac{1}{\varphi_{n}} \exp \left[-\frac{1}{2} \varphi_{n}^{2}\right]-\left(\frac{2}{2 \pi}\right)^{1 / 2} e^{-c \varphi_{n}} \frac{1}{\varphi_{n}} \exp \left[-\frac{1}{2} \varphi_{n}^{2}\right] \\
& \geqq \frac{1}{2} P\left(E_{n}\right)\left(1-2 e^{-c \varphi_{n}}\right),
\end{aligned}
$$

where $\varphi_{n}$ denotes $\varphi\left(2^{p} / l\right)$. So, for a given pair $(n, h)$, we can take $c>0$ such that

$$
P\left(F_{m}(c)\right) \geqq \frac{1}{3} P\left(E_{m}\right), \quad m \geqq n,
$$


and

$$
P\left(\bigcap_{i=h}^{n}\left(E_{i}^{\prime} \cap E_{i}(c)\right)\right) \geqq \frac{1}{2} P\left(\bigcap_{i=h}^{n} E_{i}^{\prime}\right) .
$$

Then it holds that

$$
\begin{aligned}
P\left(E_{m} / E_{h}^{\prime} \cap E_{h+1}^{\prime} \cap \cdots \cap E_{n}^{\prime}\right) & \\
& =P\left(E_{m} \cap E_{h}^{\prime} \cap E_{h+1}^{\prime} \cap \cdots \cap E_{n}^{\prime}\right) / P\left(E_{h}^{\prime} \cap E_{h+1}^{\prime} \cap \cdots \cap E_{n}^{\prime}\right) \\
& \geqq \frac{1}{2} P\left(F_{m}(c) / \bigcap_{i=h}^{n}\left(E_{i}^{\prime} \cap E_{i}(c)\right)\right) .
\end{aligned}
$$

On the other hand, if we put

$$
X_{i}=U_{h+i}, \quad A_{i}=\left[-c, \varphi\left(2^{p_{i}} / l_{i}\right)\right], \quad i=0,1,2, \ldots, n-h,
$$

and

$$
Y_{m}=U_{m}, \quad B_{m}=\left[\varphi\left(2^{p_{m}} / l_{m}\right), \varphi\left(2^{p_{m}} / l_{m}\right)+c\right], \quad m=n+1, n+2, \ldots,
$$

then, applying Lemma 9, we have for large $m$

$$
P\left(F_{m}(c) / \bigcap_{i=h}^{n}\left(E_{i}^{\prime} \cap E_{i}(c)\right)\right)>\frac{1}{2} P\left(F_{m}(c)\right) .
$$

Therefore (35) and (36) show that there exists an $H(n, h)>n$ such that

$$
P\left(E_{m} / E_{h}^{\prime} \cap E_{h+1}^{\prime} \cap \cdots \cap E_{n}^{\prime}\right) \geqq P\left(E_{m}\right) / 12, \quad m \geqq H(n, h),
$$

which proves Lemma 9 for $C(h)=1 / 12$. Q.E.D.

The proof of Lemma 8 is complicated and we need some lemmas for it.

Lemma 10. Let $(U, V)$ be a two dimensional Gaussian random variable with $E(U)=E(V)=0$ and $E\left(U^{2}\right)=E\left(V^{2}\right)=1$. Then for any $a, b>0$, there exist positive constants $K$ and $d$ such that (i)

$$
P(U>a, V>a) \leqq K \exp \left[-d\left(1-\rho^{2}\right) a^{2}\right] P(U>a),
$$

where $\rho$ denotes $\rho(U, V)$, and (ii) if $\rho<1 / a b$, then

$$
P(U>a, V>b) \leqq K P(U>a) P(V>b) .
$$

Proof. See Lemmas 3 and 4 of Chung-Erdös-Sirao [3].

Now, fur each $E_{j}=E(p ; k, l)$, let $E_{j}$ be the collection of $E_{n}=E\left(p^{\prime} ; k^{\prime}, l^{\prime}\right)$ such that $n>j$ and

$$
\rho\left(U_{j}, U_{n}\right) \geqq\left\{\varphi\left(2^{p} / l\right) \varphi\left(2^{p^{\prime}} \mid l^{\prime}\right)\right\}^{-1}
$$

Then we have

LEMma 11. For each $E_{j}=E(p ; k, l), E_{j}$ is a finite set. More precisely, there exists an absolute constant $C_{11}$ such that for $E_{n}=E\left(p^{\prime} ; k^{\prime}, l^{\prime}\right) \in E_{j}$

$$
p^{\prime}<p+C_{11} \log p \text {. }
$$


Proof. Let us set $a=k / 2^{p}, b=(k+l) / 2^{p}, c=k^{\prime} / 2^{p^{\prime}}$ and $d=\left(k^{\prime}+l^{\prime}\right) / 2^{p^{\prime}}$. Since $\rho$ is convex, we can see from Lemma 4 that $\rho\left(U_{j}, U_{n}\right) \leqq 0$ if the intervals $(a, b)$ and $(c, d)$ are disjoint. So each $E_{n} \in E_{j}$ should satisfy either one of the following:

$(\alpha) a \leqq c \leqq b \leqq d$,

( $\beta$ ) $a \leqq c \leqq d \leqq b$,

$(\gamma) c \leqq a \leqq d \leqq b$.

In the case ( $\alpha$ ), using (21) again, we have

$$
E((x(b)-x(a))(x(d)-x(c))) \leqq \sigma^{2}(b-c) .
$$

Hence the monotonicity of $\sigma^{2}$ implies

$$
\rho\left(U_{j}, U_{n}\right) \leqq \frac{\sigma^{2}(b-c)}{\sigma(b-a) \sigma(d-c)} \leqq \frac{\sigma(d-c)}{\sigma(b-a)} .
$$

Similar computation in the cases $(\beta)$ and $(\gamma)$ shows that in all cases $(\alpha),(\beta),(\gamma)$, we have

$$
\rho\left(U_{j}, U_{n}\right) \leqq \sigma(d-c) / \sigma(b-a), \quad E_{n} \in E_{j} .
$$

Combining this with (39), we have

$$
\left\{\varphi\left(2^{p} / l\right) \varphi\left(2^{p^{\prime}} / l^{\prime}\right)\right\}^{-1} \leqq \sigma\left(l^{\prime} / 2^{p^{\prime}}\right) / \sigma\left(l / 2^{p}\right) .
$$

Therefore we can see from (8) and (A.1) that there exists an absolute constant $L>0$ such that

$$
\frac{\left(p^{\prime} \mid p\right)^{1-\beta}}{2^{\left(p^{\prime}-p\right) \alpha}}>L\left(\frac{1}{p p^{\prime}}\right)^{1 / 2}
$$

or

$$
p^{\prime}+\frac{2 \beta-3}{2 \alpha \log 2} \log p^{\prime}<p+\frac{1}{2 \alpha \log 2}\{(2 \beta-1) \log p-\log L\},
$$

which implies the existence of $C_{11}$ satisfying (40). Q.E.D.

Next, let us put

$$
\begin{aligned}
E_{j} & =\left\{E_{j_{i}} ; i=1,2, \ldots, s\right\}, \\
E_{j} & =E(p ; k, l), \quad E_{j_{i}}=E\left(p_{i} ; k_{i}, l_{i}\right), \\
a & =k / 2^{p}, \quad b=(k+l) / 2^{p}, \quad a_{i}=k_{i} / 2^{p_{i}}, \quad b_{i}=\left(k_{i}+l_{i}\right) / 2^{p_{i}}, \\
\varphi & =\varphi(1 /(b-a)), \quad \varphi_{i}=\varphi\left(1 /\left(b_{i}-a_{i}\right)\right) .
\end{aligned}
$$

Then it holds that $b_{i}-a_{i} \leqq b-a, \varphi \leqq \varphi_{i}, i=1,2, \ldots, s$, because $j_{i}>j$.

Now it follows from Lemma 10, (i) that

$$
\begin{aligned}
P\left(E_{j} \cap E_{f_{l}}\right) & \leqq P\left(U_{j} \geqq \sigma(b-a) \varphi, U_{j_{i}} \geqq \sigma\left(b_{i}-a_{i}\right) \varphi\right) \\
& \leqq K \exp \left[-d\left(1-\rho_{i}^{2}\right) \varphi^{2}\right] P\left(E_{j}\right),
\end{aligned}
$$


where $\rho_{i}$ denotes $\rho\left(U_{j}, U_{j_{i}}\right)$. Hence, for the estimation of the left-hand side of (32), it suffices to do

$$
\sum_{i=1}^{s} \exp \left[-d\left(1-\rho_{i}^{2}\right) \varphi^{2}\right]
$$

To do this, we divide the above summation into two parts denoted by $\Sigma^{(1)}$ and $\sum^{(2)}$ as follows:

(44) $\sum_{i=1}^{s} \exp \left[-d\left(1-\rho_{i}^{2}\right) \varphi^{2}\right]=\sum^{(1)} \exp \left[-d\left(1-\rho_{i}^{2}\right) \varphi^{2}\right]+\sum^{(2)} \exp \left[-d\left(1-\rho_{i}^{2}\right) \varphi^{2}\right]$, where $\Sigma^{(1)}$ expresses the summation over all $i$ 's such that

$$
\rho_{i} \geqq\left(1-p^{-1 / 2}\right)^{1 / 2},
$$

and $\Sigma^{(2)}$ expresses the summation of remainder.

LEMMA 12. There exists an absolute constant $C_{12}$ such that

$$
\sum^{(2)} \exp \left[-d\left(1-\rho_{i}^{2}\right) \varphi^{2}\right] \leqq C_{12} \text {. }
$$

Proof. It suffices to prove the boundedness of $\Sigma^{(2)}$ for large $p$. So we may assume that

$$
p \log 2-(1 / \alpha) \log p>p / 2 \text {. }
$$

Since we have for all $i$ considered in $\sum^{(2)} 1-\rho_{i}^{2}>p^{-1 / 2}$, it follows from (8) that

$$
\left(1-\rho_{i}^{2}\right) \varphi^{2}>p^{-1 / 2}\{p \log 2-(1 / \alpha) \log p\}>\frac{1}{2} p^{1 / 2} .
$$

Then Lemma 4, (i) shows

$$
(a, b) \cap\left(a_{i}, b_{i}\right) \neq \varnothing\left({ }^{14}\right) .
$$

Now let $\#\left(p^{\prime}\right)$ be the number of $i$ which is considered in $\Sigma^{(2)}$ and satisfies the relation $p_{i}=p^{\prime}$. Using (48) and Lemma $11, \#\left(p^{\prime}\right)$ is estimated as follows.

$$
\begin{aligned}
\#\left(p^{\prime}\right) & <(b-a) 2^{p^{\prime}}\left(p^{\prime}\right)^{1 / \alpha} \\
& \leqq\left(p p^{\prime}\right)^{1 / \alpha} 2^{p^{\prime}-p} \\
& <p^{C_{11}+2 / \alpha}\left(1+C_{11} \log p / p\right)^{1 / \alpha}
\end{aligned}
$$

Combine this with (40) and (47), and we can see the existence of an absolute constant $\bar{C}_{12}$ such that

$$
\begin{aligned}
& \sum^{(2)} \exp \left[-d\left(1-\rho_{i}^{2}\right) \varphi^{2}\right]<p^{C_{11}+2 / \alpha}\left(1+C_{11} \log p / p\right)^{1 / \alpha} C_{11} \log p \exp \left[-\frac{1}{2} d p^{1 / 2}\right] \\
& \leqq C_{12} \text {. }
\end{aligned}
$$

LEMMA 13. There exists an absolute constant $C_{13}$ such that

$$
\sum^{(1)} \exp \left[-d\left(1-\rho_{i}^{2}\right) \varphi^{2}\right] \leqq C_{13} \text {. }
$$

(14) $\varnothing$ expresses the empty set. 
Proof. The proof is divided into several steps.

Using the notations given in (42), we first note that there exist three cases:

( $\alpha) a \leqq a_{i} \leqq b \leqq b_{i}$,

( $\beta) ~ a \leqq a_{i} \leqq b_{i} \leqq b$,

( $\gamma) a_{i} \leqq a \leqq b_{i} \leqq b$.

$1^{\circ}$. There exists an absolute constant $C_{14}$ such that for all $p_{i}$ 's considered in $\sum^{(1)}$

$$
p \leqq p_{i} \leqq p+C_{14}
$$

In fact, as was shown in (41) already, $\rho_{i}=\rho\left(U_{j}, U_{j_{i}}\right)$ satisfies $\rho_{i} \leqq \sigma\left(b_{i}-a_{i}\right) / \sigma(b-a)$. And accordingly we can see by (45) and (A.1) that for large $p$

$$
1-p^{-1 / 2} \leqq \rho_{i}^{2}<2 \frac{C_{4}}{C_{3}}\left(\frac{3}{2}\right)^{\alpha} 2^{-\left(p_{i}-p\right) \alpha}\left(\frac{p_{i}}{p}\right)^{1-\beta}
$$

Then Lemma 11 shows the existence of $C_{15}$ such that

$$
1-p^{-1 / 2} \leqq C_{15} 2^{-\left(p_{i}-p\right) \alpha}
$$

which implies (50).

$2^{\circ}$. In the case $(\alpha)$, there exists an absolute constant $C_{16}$ such that

$$
\rho_{i} \leqq 1-C_{16}\left(k_{i}-k 2^{p_{i}-p}\right)^{\alpha} / p .
$$

To prove this, we first remark that (41) and (45) imply

$$
\lim _{p \rightarrow \infty} \frac{\sigma\left(b-a_{i}\right)}{\sigma(b-a)}=\lim _{p \rightarrow \infty} \frac{\sigma\left(b_{i}-a_{i}\right)}{\sigma(b-a)}=1 .
$$

Then we have

$$
\lim _{p \rightarrow \infty} \frac{\sigma\left(a_{i}-a\right)}{\sigma(b-a)}=\lim _{p \rightarrow \infty} \frac{\sigma\left(a_{i}-a\right)}{\sigma\left(b-a_{i}\right)}=0
$$

because we have by Lemma 4, (i)

$$
\begin{aligned}
\rho_{i} & \leqq \frac{E\left((x(b)-x(a))\left(x(b)-x\left(a_{i}\right)\right)\right)}{\sigma(b-a) \sigma\left(b_{i}-a_{i}\right)} \\
& =\frac{\sigma^{2}(b-a)+\sigma^{2}\left(b-a_{i}\right)-\sigma^{2}\left(a_{i}-a\right)}{2 \sigma(b-a) \sigma\left(b_{i}-a_{i}\right)}
\end{aligned}
$$

and $\rho_{i} \rightarrow 1$ as $p \rightarrow \infty$. Moreover we can see from (50) that for any $\varepsilon>0$ there exists $p(\varepsilon)$ such that

$$
1 \leqq\left|\frac{\log \left(a_{i}-a\right)}{\log (b-a)}\right|=\left|\frac{p_{i} \log 2-\log \left(k_{i}-k 2^{p_{i}-p}\right)}{p \log 2-\log l}\right|<1+\varepsilon, \quad p \geqq p(\varepsilon),
$$

because $a_{i}>a$ in the case $(\alpha)$. 
Now, if we remark that for large $p$

$$
\begin{aligned}
\sigma^{2}\left(a_{i}-a\right)-\{\sigma(b-a) & \left.-\sigma\left(b-a_{i}\right)\right\}^{2} \\
& \geqq \sigma^{2}\left(a_{i}-a\right)-\left\{\left(\sigma^{2}\left(a_{i}-a\right)+\sigma^{2}\left(b-a_{i}\right)\right)^{1 / 2}-\sigma\left(b-a_{i}\right)\right\}^{2}\left(^{15}\right) \\
& \geqq \sigma^{2}\left(a_{i}-a\right)-\sigma^{2}\left(b-a_{i}\right)\left\{\left(1+\sigma^{2}\left(a_{i}-a\right) / \sigma^{2}\left(b-a_{i}\right)\right)^{1 / 2}-1\right\}^{2} \\
& \geqq \sigma^{2}\left(a_{i}-a\right) / 2, \quad(\text { by }(52)),
\end{aligned}
$$

it follows from (53), (54), and (A.1) that for large $p$

$$
\begin{aligned}
\rho_{i} & \leqq \frac{2 \sigma(b-a) \sigma\left(b-a_{i}\right)+\left\{\sigma(b-a)-\sigma\left(b-a_{i}\right)\right\}^{2}-\sigma^{2}\left(a_{i}-a\right)}{2 \sigma(b-a) \sigma\left(b_{i}-a_{i}\right)} \\
& \leqq 1-\frac{1}{4} \frac{\sigma^{2}\left(a_{i}-a\right)}{\sigma^{2}(b-a)} \\
& \leqq 1-\frac{1}{4}(1+\varepsilon)^{-|\beta|} \frac{C_{3}}{C_{4}}\left(a_{i}-a\right)^{\alpha}(b-a)^{-\alpha} \\
& \leqq 1-\frac{1}{4}(1+\varepsilon)^{-|\beta|} \frac{C_{3}}{C_{4}}\left(k_{i}-k 2^{p_{i}-p}\right)^{\alpha} l^{-\alpha} 2^{-\left(p_{i}-p\right) \alpha} \\
& \leqq 1-\frac{1}{4}(1+\varepsilon)^{-|\beta|} \frac{C_{3}}{C_{4}}\left(k_{i}-2 k^{p_{i}-p}\right)^{\alpha} p^{-1} 2^{-\left(p_{i}-p\right) \alpha} .
\end{aligned}
$$

Then (50) implies (51).

$3^{\circ}$. In the case $(\gamma)$, we have similarly as in $2^{\circ}$

$$
\rho_{i} \leqq 1-C_{17}\left\{(k+l) 2^{p_{i}-p}-\left(k_{i}+l_{i}\right)\right\}^{\alpha} / p,
$$

where $C_{17}$ is an absolute constant.

$4^{\circ}$. In the case $(\beta)$, we have by Lemma 4 , (i) $\rho_{i} \leqq \rho\left(x(b)-x\left(a_{i}\right), x\left(b_{i}\right)-x\left(a_{i}\right)\right)$. So, if we put $k 2^{p_{i}-p}=k_{i}$ in (55), it follows from (55) that

$$
\rho_{i} \leqq 1-C_{17}\left(l 2^{p_{i}-p}-l_{i}\right)^{\alpha} / p .
$$

$5^{\circ}$. Let us divide the summation $\sum^{(1)}$ into three parts as follows:

$$
\sum^{(1)}=\sum^{(\alpha)}+\sum^{(\beta)}+\sum^{(\gamma)}
$$

where $\Sigma^{(\alpha)}, \Sigma^{(\beta)}$ and $\Sigma^{(\gamma)}$ denote the summations over all $i$ 's corresponding to the cases $(\alpha),(\beta),(\gamma)$ respectively. Then we can see from (8), (51), (55) and (56) that there exists an absolute constant $d_{1}>0$ such that

$$
\begin{aligned}
d\left(1-\rho_{i}^{2}\right) \varphi^{2} & \geqq d_{1}\left(k_{i}-2^{p_{i}-p} k\right)^{\alpha}, \quad \text { for the case }(\alpha), \\
& \geqq d_{1}\left(l 2^{p_{i}-p}-l_{i}\right)^{\alpha}, \quad \text { for the case }(\beta), \\
& \geqq d_{1}\left((k+l) 2^{p_{i}-p}-\left(k_{i}+l_{i}\right)\right)^{\alpha}, \quad \text { for the case }(\gamma) .
\end{aligned}
$$

$\left({ }^{15}\right) \sigma^{2}$ is concave. 
Next, for fixed $p^{\prime}(\geqq p)$ and $k^{\prime}$, we consider the numbers $\#\left(p^{\prime}, k^{\prime} ; \alpha\right)$ of $i$ which corresponds to the case $(\alpha)$ and satisfies the relations $p_{i}=p^{\prime}$ and $k_{i}=k^{\prime}$. Then we have $\#\left(p^{\prime}, k^{\prime} ; \alpha\right) \leqq k^{\prime}-k 2^{p^{\prime}-p}$.

Further, for given $p^{\prime}, l^{\prime}, h^{\prime}$, let us set

$\#\left(p^{\prime}, l^{\prime} ; \beta\right)=$ the number of $i$ which corresponds to the case $(\gamma)$ and satisfies the conditions $p_{i}=p^{\prime}$ and $l_{i}=l^{\prime}$.

$\#\left(p^{\prime}, h^{\prime} ; \gamma\right)=$ the number of $i$ which corresponds to the case $(\gamma)$ and satisfies the conditions $p_{i}=p^{\prime}$ and $k_{i}+l_{i}=h^{\prime}$.

Then it holds that $\#\left(p^{\prime}, l^{\prime} ; \beta\right) \leqq l 2^{p^{\prime}-p}-l^{\prime}, \#\left(p^{\prime}, h^{\prime} ; \gamma\right) \leqq(k+l) 2^{p^{\prime}-p}-h^{\prime}$.

Now we have by (50) and (57)

$$
\begin{aligned}
& \sum^{(1)} \exp \left[-d\left(1-\rho_{i}^{2}\right) \varphi^{2}\right] \\
& \qquad \sum_{p^{\prime}=p}^{p+C_{14}}\left\{\sum_{k^{\prime}=k 2^{p^{\prime}-p}}^{(k+l) 2^{p^{\prime}-p}}\left(k^{\prime}-k 2^{p^{\prime}-p}\right) \exp \left[-d_{1}\left(k^{\prime}-2 k^{p^{\prime}-p}\right)^{\alpha}\right]\right. \\
& \quad+\sum_{l^{\prime}=0}^{l 2^{p^{\prime}-p}}\left(l 2^{p^{\prime}-p}-l^{\prime}\right) \exp \left[-d_{1}\left(l 2^{p^{\prime}-p}-l^{\prime}\right)^{\alpha}\right] \\
& \left.\quad+\sum_{h^{\prime}=k 2^{p^{\prime}-p}}^{(k+l)^{p^{\prime}-p}}\left((k+l) 2^{p^{\prime}-p}-h^{\prime}\right) \exp \left[-d_{1}\left((k+l) 2^{p^{\prime}-p}-h^{\prime}\right)^{\alpha}\right]\right\} \\
& <3 C_{14} \sum_{k=0}^{\infty} k \exp \left[-d_{1} k^{\alpha}\right] \\
& <\infty,
\end{aligned}
$$

which proves (49). Q.E.D.

Now we shall prove Lemma 8.

Proof of Lemma 8. For any $E_{j}$, let us take $E_{j}$ (the collection of $E_{n}$ satisfying (39)) as the set $\left\{E_{j_{i}} ; i=1,2, \ldots, s\right\}$ in Lemma 8 . Since (39) and Lemma 10 imply (33), it suffices to prove the validity of (32).

According to (43), we have

$$
\sum_{i=1}^{s} P\left(E_{j} \cap E_{j_{i}}\right) \leqq K \sum_{i=1}^{s} \exp \left[-d\left(1-\rho_{i}^{2}\right) \varphi^{2}\right] P\left(E_{j}\right) .
$$

Then we get from (44), Lemmas 12 and 13

$$
\sum_{i=1}^{s} P\left(E_{j} \cap E_{j_{i}}\right) \leqq K\left(C_{12}+C_{13}\right) P\left(E_{j}\right) .
$$

This shows that (32) holds for $K_{1}=K\left(C_{12}+C_{13}\right)$.

Thus we have completed the proof of Theorem 3. Q.E.D.

6. Proof of Theorem 4. The proof of Theorem 4 proceeds parallel with the one of Theorem 2.

LEMMA 14. Theorems 4 and 5 hold if they do under the following condition:

$$
\left\{2 \log _{(2)} t\right\}^{1 / 2} \leqq \psi(t) \leqq\left\{3 \log _{(2)} t\right\}^{1 / 2}, \quad t \geqq e^{e} \vee a .
$$


The proof is analogous to the one of Lemma 6 in T. Sirao [7], and we omit it. In the following discussion, we shall always assume (58). Let us define $E(p ; k)$ by

$$
\begin{aligned}
E(p ; k)=\left\{\omega ; x\left(k / 2^{p}\right)-x(0)\right. & \left.\geqq \sigma\left(k / 2^{p}\right) \psi\left(2^{p} / k\right)\right\}, \\
& p=1,2,3, \ldots, k=1,2,3, \ldots, 2^{p} .
\end{aligned}
$$

Then we have the following one corresponding to Lemma 2.

Lemma 15. For any $c \in(0,1)$,

$$
\sum_{p=1}^{\infty} \sum_{k=\left[c(\log p)^{1 / \alpha}\right]}^{\left[(\log p)^{1 / \alpha}\right]} P(E(p ; k))
$$

converges or diverges according as

$$
\int_{a}^{\infty} \frac{1}{t} \psi(t)^{2 / \alpha-1} \exp \left[-\frac{1}{2} \psi^{2}(t)\right] d t
$$

converges or diverges.

Proof. By the similar way as in the proof of Lemma 2, we can see that there exist $p_{0}$ and $p_{1}$ such that

$$
\begin{aligned}
\sum_{p=p_{0}}^{\infty} \sum_{k=\left[c(\log p)^{1 / \alpha}\right]+1}^{\left[(\log p)^{1 / \alpha}\right]} P(E(p ; k)) & \leqq \frac{2}{(2 \pi)^{1 / 2}} \sum_{p=p_{0}}^{\infty}\left\{\frac{2^{p}}{(\log p)^{1 / \alpha}}-\frac{2^{p-1}}{(\log (p-1))^{1 / \alpha}}\right\} \frac{(\log p)^{2 / \alpha}}{2^{p}} \\
& \frac{1}{\psi\left(2^{p}(\log p)^{-1 / \alpha}\right)} \exp \left[-\frac{1}{2} \psi^{2}\left(2^{p}(\log p)^{-1 / \alpha}\right)\right] \\
& \leqq \frac{2}{(2 \pi)^{1 / 2}} \sum_{p=p_{0}}^{\infty} \int_{2^{p-1}(\log (p-1))^{-1 / \alpha}}^{2^{p(\log p)-1 / \alpha}} \frac{(\log p)^{1 / \alpha}}{2^{p}}\left\{\psi\left(2^{p}(\log p)^{-1 / \alpha}\right)\right\}^{2 / \alpha-1} \\
& \leqq \frac{2}{(2 \pi)^{1 / 2}} \int_{a}^{\infty} \frac{1}{t} \psi(t)^{2 / \alpha-1} \exp \left[-\frac{1}{2} \psi^{2}\left(2^{p}(\log p)^{-1 / \alpha}\right)\right] d t
\end{aligned}
$$

and similarly

$$
\sum_{p=p_{1}}^{\infty} \sum_{k=\left[c(\log p)^{1 / \alpha}\right]}^{\left[(\log p)^{1 / \alpha}\right]} P(E(p ; k)) \geqq \frac{1}{2(2 \pi)^{1 / 2}} \int_{a / c}^{\infty} \frac{1}{t} \psi(t)^{2 / \alpha-1} \exp \left[-\psi^{2}(t)\right] d t
$$

These two inequalities prove the lemma. Q.E.D.

According to Lemma 15, it holds that under the assumption (4)

$$
\sum_{p=1}^{\infty} \sum_{k=\left[(\log p)^{1 / \alpha / 3}\right]}^{\left[(\log p)^{1 / \alpha}\right]} P(E(p ; k))<\infty .
$$

Now, for a given pair of $(p, k)$, we set

$$
b=k 2^{-p}, \quad h_{q}=2^{-p} \exp [-q c], \quad \psi \equiv \psi(1 / b), \quad q=1,2,3, \ldots,
$$


where $c$ is a large number which makes $e^{c}$ an integer and satisfies the following inequalities where absolute constants $C_{18}$ and $C_{19}$ will be chosen later.

$$
\begin{gathered}
\left(\frac{2}{\pi}\right)^{1 / 2} \frac{1}{C_{18}} \exp \left[-\frac{C_{18}^{2}}{8}\left(\frac{e}{4}\right)^{\alpha(q-1) c}\right]<e^{-2 q c}, \quad q \geqq 2, \\
C_{18}\left(e^{c / 4} / 2\right)^{\alpha(q-1)}>2 C_{19} .
\end{gathered}
$$

Then the set $S_{q}$ and the events $F(q ; m), F(q)$ are defined by $S_{q}=\left\{m ; 0 \leqq m \leqq e^{q c}\right\}$, $q \geqq 1$,

$$
F(q ; m)=\left\{\omega ; x\left(b-m h_{q}\right)-x(0)>\sigma\left(b-m h_{q}\right)\left\{\psi+\frac{2 c}{\psi} \sum_{i=0}^{q-1} 2^{-\alpha i}\right\}\right\}
$$

and $F_{q}=\bigcup_{m \in S_{q}} F(q ; m)$.

$$
q \geqq 1, m \in S_{q} \text {, }
$$

LemMa 16. There exists an absolute constant $C_{20}$ such that

$$
P\left(\bigcup_{q=1}^{\infty} F_{q}\right) \leqq C_{20} P(E(p ; k))
$$

Proof. For any $m \in S_{q}, q \geqq 2$, we choose $m_{1} \in S_{q-1}$ such that $\left|m h_{q}-m_{1} h_{q-1}\right|$ $\leqq h_{q-1}$. And we set $U_{m}=x\left(b-m h_{q}\right)-x(0), V_{m_{1}}=x\left(b-m_{1} h_{q-1}\right)-x(0)$. Then we have by Schwartz's inequality and (A.1).

$$
\begin{aligned}
\rho\left(U_{m}, V_{m_{1}}\right) & =\frac{\sigma^{2}\left(b-m h_{q}\right)+\sigma^{2}\left(b-m_{1} h_{q-1}\right)-\sigma^{2}\left(m h_{q}-m_{1} h_{q-1}\right)}{2 \sigma\left(b-m h_{q}\right) \sigma\left(b-m_{1} h_{q-1}\right)} \\
& \geqq 1-\frac{1}{2} \frac{\sigma^{2}\left(m h_{q}-m_{1} h_{q-1}\right)}{\sigma\left(b-m h_{q}\right) \sigma\left(b-m_{1} h_{q-1}\right)} .
\end{aligned}
$$

Moreover we can see, by the same way as in the proof of Lemma 6 , that there exists an absolute constant $M$ such that

$$
\rho\left(U_{m}, V_{m_{1}}\right) \geqq 1-M \frac{1}{\log p} \exp \left[-\frac{(q-1) c}{2} \alpha\right]=\rho_{0} \text { (say). }
$$

Now put

$$
C_{19}=\sup \left\{\left(1-\rho_{0}^{2}\right)^{-1 / 2}\left(1-\rho_{0}\right)\left(\psi+\frac{2 c}{\psi} \sum_{i=0}^{q-2} 2^{-\alpha i}\right) ; p \geqq e^{M} \vee e, q \geqq 2\right\},
$$

and choose $C_{18}$ which satisfies (60) and the following

$$
\left(1-\rho_{0}^{2}\right)^{-1 / 2} \rho_{0} \frac{2 c}{\varphi} \geqq C_{18} \exp \left[\frac{1}{4} \alpha(q-1) c\right], \quad p \geqq e^{M}, q \geqq 2 .
$$

(Evidently we can choose such triple $\left(c, C_{18}, C_{19}\right)$ if we take sufficiently large $c$.) Then we have by the procedure as was used in Lemma 6

$$
P\left(F\left(q-1 ; m_{1}\right)^{\prime} \cap F(q ; m)\right) \leqq e^{-2 q c} P(E(p ; k)),
$$


and accordingly

$$
\begin{aligned}
P\left(F_{q}\right) & \leqq P\left(F_{q-1}\right)+\sum_{m \in S_{q}} P\left(F_{q-1}^{\prime} \cap F(q ; m)\right) \\
& \leqq P\left(F_{q-1}\right)+e^{-q c} P(E(p ; k)), \quad q \geqq 2 .
\end{aligned}
$$

Since $P\left(F_{1}\right) \leqq e^{c} P(E(p ; k)),(61)$ proves the lemma. Q.E.D.

Next, let $H(p ; k)$ be the collection of $\omega$ such that there exists $t$ satisfying $(k-1) / 2^{p}$ $\leqq t<k / 2^{p}$ and

$$
x(t, \omega)-x(0, \omega) \geqq \sigma(t)\left\{\psi\left(2^{p} / k\right)+2 c\left(\psi\left(2^{p} / k\right)\right)^{-1} \sum_{i=0}^{\infty} 2^{-\alpha i}\right\}
$$

Then we can see by Lemma 16 and the continuity of $X$

$$
P(H(p ; k)) \leqq \liminf _{q \rightarrow \infty} P\left(F_{q}\right) \leqq C_{20} P(E(p ; k)),
$$

and accordingly

$$
\sum_{p=p_{0}}^{\infty} \sum_{k=\left[(\log p)^{1 / \alpha} / 3\right]}^{\left[(\log p)^{1 / \alpha}\right]} P(H(p ; k))<\infty
$$

where $p_{0}=\left[e^{M} \vee e\right]+1$. Now the procedure used in the proof of Theorem 2 implies Theorem 4. Q.E.D.

7. Proof of Theorem 5. We have by Lemma 15

$$
\sum_{p=p_{0}}^{\infty} \sum_{k=\left[(\log p)^{1 / \alpha} / 3\right]}^{\left[(\log p)^{1 / \alpha}\right]} P(E(p ; k))=\infty,
$$

where $p_{0}$ denotes a sufficiently large integer so that for $p \geqq p_{0}$, we can do all the computations in the sequel which are available for large $p\left({ }^{16}\right)$.

As in $\$ 4$, we denote $E(p ; k)$ by $E_{n}$, where the subscript $n$ is given as follows: If $E_{n}=E(p ; k)$ and $E_{m}=E\left(p^{\prime} ; k^{\prime}\right)$, then $n<m$ if and only if either one of the following holds:

(i) $p<p^{\prime}$,

(ii) $p=p^{\prime}$ and $k^{\prime}<k$.

So $n<m$ implies $k^{\prime} / 2^{p^{\prime}} \leqq k / 2^{p}$.

Now it suffices to show that Lemmas 7 and 8 hold for our sequence

$$
\left\{E_{j} ; j=1,2,3, \ldots\right\} \text {. }
$$

For $E_{n}=E(p ; k)$ and $E_{m}=E\left(p^{\prime} ; k^{\prime}\right)$, put $b=k / 2^{p}, b^{\prime}=k^{\prime} / 2^{p^{\prime}}, \quad U_{n}=x(b)-x(0)$, $U_{m}=x\left(b^{\prime}\right)-x(0)$.

Then we have by (34) $\lim _{m \rightarrow \infty} \rho\left(U_{n}, U_{m}\right)=0$, or more precisely

$$
\rho\left(U_{n}, U_{m}\right) \leqq \sigma\left(b^{\prime}\right) / \sigma(b)
$$

${ }^{(16)}$ This assumption does not take any loss of generality. 
as was obtained in $\S 3$. Therefore the proof of Lemma 7 is valid for the present case if we replace there $\varphi$ and $l / 2^{p}$ by $\psi$ and $k / 2^{p}$ respectively, i.e. Lemma 7 holds for our sequence $\left\{E_{j} ; j=1,2,3, \ldots\right\}$.

Next, we shall consider Lemma 8. For each $E_{j}=E(p ; k)$, let $\boldsymbol{E}_{j}$ be the collection of events $E_{n}=E\left(p^{\prime} ; k^{\prime}\right)$ such that $n>j$ and

$$
\rho\left(U_{j}, U_{n}\right) \geqq\left\{\psi\left(2^{p} / k\right) \psi\left(2^{p^{\prime}} \mid k^{\prime}\right)\right\}^{-1} .
$$

Then we have

LEMMA 17. For each $E_{f}=E(p ; k), E_{f}$ is a finite set. More precisely, there exists an absolute constant $C_{21}$ such that for $E_{n}=E\left(p^{\prime} ; k^{\prime}\right) \in E_{j}$

$$
p^{\prime}<p+C_{21} \log \log p \text {. }
$$

Proof. Since we have for $E_{n} \in E_{j}$

$$
\{\psi(a) \psi(b)\}^{-1} \leqq \rho\left(U_{j}, U_{n}\right) \leqq \sigma\left(b^{\prime}\right) / \sigma(b)
$$

where $b=k / 2^{p}$ and $b^{\prime}=k^{\prime} / 2^{p^{\prime}}$, it follows by (A.1) that there exists an absolute constant $L>0$ such that

$$
\frac{1}{2^{\left(p^{\prime}-p\right) \alpha}}\left(\frac{\log p^{\prime}}{\log p}\right)^{\alpha}\left(\frac{p}{p^{\prime}}\right)^{\beta} \geqq \frac{L}{\log p \log p^{\prime}}
$$

which proves Lemma 17. Q.E.D.

Now let

$$
\begin{aligned}
& E_{j}=\left\{E_{j_{i}} ; i=1,2, \ldots, s\right\}, \quad E_{j}=E(p ; k), E_{j_{i}}=E\left(p_{i} ; k_{i}\right), \\
& b=k / 2^{p}, \quad b_{i}=k_{i} / 2^{p_{i}}, \quad \psi=\psi(1 / b), \quad \text { and } \quad \rho_{i}=\rho\left(U_{j}, U_{j_{i}}\right) .
\end{aligned}
$$

According to Lemma 10 , the validity of Lemma 8 for our sequence

$$
\left\{E_{j} ; j=1,2,3, \ldots\right\}
$$

is obtained from the boundedness of

$$
\sum_{i=1}^{s} \exp \left[-d\left(1-\rho_{i}^{2}\right) \psi^{2}\right]
$$

where $d$ denotes an absolute positive constant. To show the boundedness of the above series, we divide it into two parts as follows:

$$
\sum_{i=1}^{s} \exp \left[-d\left(1-\rho_{i}^{2}\right) \psi^{2}\right]=\sum^{(1)} \exp \left[-d\left(1-\rho_{i}^{2}\right) \psi^{2}\right]+\sum^{(2)} \exp \left[-d\left(1-\rho_{i}^{2}\right) \psi^{2}\right],
$$

where $\Sigma^{(1)}$ expresses the summation over all $i$ 's such that $\rho_{i} \geqq\left(1-(\log p)^{-1}\right)^{1 / 2}$ and $\Sigma^{(2)}$ does the summation of remainder. Then we have

Lemma 18. There exists an absolute constant $C_{22}$ such that

$$
\sum^{(2)} \exp \left[-d\left(1-\rho_{i}^{2}\right) \psi^{2}\right]<C_{22} \text {. }
$$


Proof. Since it follows from the definition of $\sum^{(2)}$ that for any $i$ considered in $\sum^{(2)}, 1-\rho_{i}^{2}>(\log p)^{-1 / 2}$, we have by Lemma 14

$$
\left(1-\rho_{i}^{2}\right) \psi \geqq(\log p)^{1 / 2} \log p \geqq(\log p)^{1 / 2} .
$$

On the other hand, the cardinal number of collection of $k^{\prime}$ satisfying $k^{\prime} / 2^{p^{\prime}}$ $\leqq k / 2^{p}$ for previously given $p^{\prime}$ does not exceed $2^{p^{\prime}-p}(\log p)^{1 / \alpha}$. Moreover we can see by Lemma 17

Then we have

$$
2^{p^{\prime}-p}(\log p)^{1 / \alpha}<(\log p)^{C_{21}+1 / \alpha}
$$

$$
\sum^{(2)} \exp \left[-d\left(1-\rho_{i}^{2}\right) \psi^{2}\right] \leqq(\log p)^{C_{21}+1 / \alpha} \exp \left[-d(\log p)^{1 / 2}\right]
$$

which proves Lemma 18. Q.E.D.

Lemma 19. There exists an absolute constant $C_{23}$ such that

$$
\sum^{(1)} \exp \left[-d\left(1-\rho_{i}^{2}\right) \psi^{2}\right] \leqq C_{23} \text {. }
$$

Proof. $1^{\circ}$. For any $i$ considered in $\Sigma^{(1)}$, it holds by (62) and (A.1) that

$$
1-(\log p)^{-1 / 2} \leqq \rho_{i}^{2} \leqq \frac{\sigma^{2}\left(b_{i}\right)}{\sigma^{2}(b)} \leqq 2 \frac{C_{4}}{C_{3}} 2^{\left(p^{\prime}-p\right) \alpha} \frac{\log p}{\log p^{\prime}}\left(\frac{p}{p^{\prime}}\right)^{\beta}
$$

So we can see that there exists an absolute constant $C_{25}>0$ such that

$$
p \leqq p^{\prime}<p+C_{25} \text {. }
$$

$2^{\circ}$. Considering the special case of $(\beta)$ in the proof of Lemma 13, where $a=a_{i}=0$ and $b_{i}<b$, we can see from (56) that for a properly chosen absolute constant $C_{25} \rho_{i} \leqq 1-C_{25}\left(\left(b-b_{i}\right) / b\right)^{\alpha}$ because the term $\left(l 2^{p_{i}-p}-l_{i}\right)^{\alpha} / p$ in (56) comes from $\sigma^{2}\left(b-b_{i}\right) / \sigma^{2}(b-a)$ and now $a=0$. Therefore we have by (A.1)

or

$$
\rho_{i} \leqq 1-C_{25}\left(k 2^{p^{\prime}-p}-k^{\prime}\right)^{\alpha} / \log p
$$

$$
1-\rho_{i}^{2}>C_{25}\left(k 2^{p^{\prime}-p}-k^{\prime}\right)^{\alpha} / \log p .
$$

$3^{\circ}$. We have by (66), (67) and Lemma 14

$$
\begin{aligned}
\sum^{(1)} \exp \left[-d\left(1-\rho_{i}^{2}\right) \psi^{2}\right] & \leqq \sum^{(1)} \exp \left[-d C_{25}\left(k 2^{p^{\prime}-p}-k^{\prime}\right)^{\alpha}\right] \\
& \leqq C_{24} \sum_{k=1}^{\infty} \exp \left[-d C_{25} k^{\alpha}\right]<\infty
\end{aligned}
$$

which proves Lemma 19. Q.E.D.

Now we can get from (65), Lemmas 18 and 19 the boundedness of series in (64), as was to be proved. Q.E.D.

8. Proof of Theorem 6 and Corollary 6.1. At first we shall prove the following lemma which was stated in $\S 2$ as a sufficient condition to make $X$ a process satisfying the condition (A). 
LEMMA 20. Let $f$ be the spectral density function of stationary Gaussian process $X$. If $f$ satisfies the following conditions, then $X$ satisfies the condition (A).

(i) There exist positive constants $C_{3}, C_{4}$ and $K$ such that

$$
C_{3} \leqq f(x) x^{\alpha+1}(\log x)^{\beta} \leqq C_{4}, \quad x \geqq K,
$$

where $0<\alpha<2,-\infty<\beta<\infty$.

(ii) $g(x)=x^{2} f(x)$ is two times differentiable in $x$, and for some $0<\varepsilon<1$, either one of the following (a) or (b) holds.

(a) $x^{3-\varepsilon} g^{\prime \prime}(x)$ is bounded from below, and $\lim _{\inf _{x \rightarrow \infty}} x^{3-\varepsilon} g^{\prime \prime}(x)>0$.

(b) $x^{3-\varepsilon} g^{\prime \prime}(x)$ is bounded from above, and lim $\sup _{x \rightarrow \infty} x^{3-\varepsilon} g^{\prime \prime}(x)<0$.

Proof. For the process $X$ with a spectral density $f$ satisfying (68) and $\rho(0)=1$, we have

Then we have

$$
\begin{aligned}
\sigma^{2}(h) & =2(1-\rho(h))=4 \int_{0}^{\infty} \sin ^{2}(h x / 2) f(x) d x \\
& =4 \int_{0}^{K} \sin ^{2}(h x / 2) f(x) d x+4 \int_{K}^{\infty} \sin ^{2}(h x / 2) f(x) d x \\
& =4\left(I_{1}(h)+I_{2}(h)\right) \quad \text { (say). }
\end{aligned}
$$

$$
\lim _{h \rightarrow 0} I_{1}(h) / h^{2}=\int_{0}^{K} x^{2} f(x) d x / 4
$$

and

$$
C_{3} \int_{K}^{\infty} \sin ^{2}(h x / 2) f_{1}(x) d x \leqq I_{2}(h) \leqq C_{4} \int_{K}^{\infty} \sin ^{2}(h x / 2) f_{1}(x) d x,
$$

where $f_{1}(x)=x^{-(\alpha+1)}(\log x)^{-\beta}$. Taking a computation on the integral in (70), we have

$\int_{K}^{\infty} \sin ^{2}(h x / 2) f_{1}(x) d x=h^{\alpha}(\log 1 / h)^{-\beta} \int_{h K}^{\infty} \sin ^{2}(x / 2) x^{-(\alpha+1)}|1-(\log x / \log h)|^{-\beta} d x$, and accordingly

$$
C_{3} K_{1} \leqq \lim _{h \rightarrow 0} I_{2}(h) / h^{\alpha}(\log 1 / h)^{-\beta} \leqq C_{4} K_{1},
$$

where $K_{1}=\int_{0}^{\infty} \sin ^{2}(x / 2) x^{-(\alpha+1)} d x$.

Now (69) and (71) implies (A.1).

Next, we shall prove the convexity of $\sigma^{2}$ if (a) and (ii) hold. Let $h>0$ and consider the quantity

$$
\Delta_{h}^{(2)} \sigma^{2}=\sigma^{2}\left(h_{1}+2 h\right)+\sigma^{2}\left(h_{1}\right)-2 \sigma^{2}\left(h_{1}+h\right) .
$$

Then we have

$$
\begin{aligned}
\Delta_{h}^{(2)} \sigma^{2} & =4 \int_{0}^{\infty}\left\{\sin ^{2}\left(\left(h_{1}+2 h\right) x / 2\right)+\sin ^{2}\left(h_{1} x / 2\right)-2 \sin ^{2}\left(\left(h_{1}+h\right) x / 2\right)\right\} f(x) d x \\
& =4 \int_{0}^{\infty} \frac{\sin ^{2}(x / 2)}{x}\left\{\frac{x}{h_{1}+2 h} f\left(\frac{x}{h_{1}+2 h}\right)+\frac{x}{h} f\left(\frac{x}{h_{1}}\right)-2 \frac{x}{h_{1}+h} f\left(\frac{x}{h_{1}+h}\right)\right\} d x .
\end{aligned}
$$


Putting $\hat{f}(h ; x)=(x / h) f(x / h), g(x)=x^{2} f(x)$, we get

$$
\frac{\partial^{2} \hat{f}}{\partial h^{2}}=x h^{-3}\left\{2 f\left(\frac{x}{h}\right)+4 \frac{x}{h} f^{\prime}\left(\frac{x}{h}\right)+\left(\frac{x}{h}\right)^{2} f^{\prime \prime}\left(\frac{x}{h}\right)\right\}=x h^{-3} g^{\prime \prime}(x / h) .
$$

So it follows from (72) that

$$
\begin{aligned}
\Delta_{h}^{(2)} \sigma^{2} & =4 \int_{0}^{\infty} x^{-1} \sin ^{2}(x / 2) \Delta_{h}^{(2)} \hat{f}\left(h_{1} ; x\right) d x \\
& =4 \int_{0}^{\infty} \frac{\sin ^{2}(x / 2)}{x} \frac{h^{2} x}{\left(h_{1}+\theta h\right)^{3}} g^{\prime \prime}\left(\frac{x}{h_{1}+\theta h}\right) d x, \quad 0<\theta<2 .
\end{aligned}
$$

Then by (a) and Fatou's lemma

$$
\begin{aligned}
\liminf _{h, h_{1} \rightarrow 0} & \frac{\left(\Delta_{h}^{(2)} \sigma^{2}\right)\left(h_{1}+2 h\right)^{\varepsilon}}{4 h^{2}} \\
& \geqq \int_{0}^{\infty} x^{-(3-\varepsilon)} \sin ^{2}(x / 2) \liminf _{h, h_{1} \rightarrow 0}\left(x /\left(h_{1}+\theta h\right)\right)^{3-\varepsilon} g^{\prime \prime}\left(x /\left(x_{1}+\theta h\right)\right) d x>0,
\end{aligned}
$$

which proves the convexity of $\sigma^{2}$ in a small interval $(0, \delta)$.

If (b) holds, then we can prove the concavity of $\sigma^{2}$ in a similar way. Q.E.D.

Using Lemma 20, we can prove Theorem 6 as follows.

Proof of Theorem 6. According to the preceding lemma, there exists a stationary Gaussian process $Y=\{y(t) ; 0 \leqq t \leqq 1\}$ with 0 -mean which is independent of $X$ and satisfies the condition (A) for a given pair $(\alpha, \beta)$ with $0<\alpha<2,-\infty<\beta<\infty\left({ }^{17}\right)$. For an arbitrary $\varepsilon^{\prime}>0$, let us consider a stationary Gaussian process

$$
Z=\{z(t) ; 0 \leqq t \leqq 1\}
$$

defined by

$$
z(t)=\left(1+\varepsilon^{\prime 2}\right)^{-1 / 2}\left\{x+\varepsilon^{\prime} y(t)\right\}, \quad 0 \leqq t \leqq 1 .
$$

Then we have $E(z(t))=0, \rho_{z}(0)=E\left(z(t)^{2}\right)=1$ and for small $h>0$ and a properly chosen constant $C_{26}>0$

$$
C_{26} \frac{h^{\alpha}}{|\log h|^{\beta}} \leqq \sigma_{z}^{2}(h) \leqq \frac{\left(C_{4}+\varepsilon^{\prime 2} C_{26}\right) h^{\alpha}}{\left(1+\varepsilon^{\prime 2}\right)|\log h|^{\beta}}
$$

where

$$
\sigma_{z}^{2}(h)=E\left((z(t+h)-z(t))^{2}\right)
$$

Since the concavity or convexity of $\sigma_{z}^{2}$ is obtained from those of $\sigma^{2}$ and $\sigma_{y}^{2}$, where $\sigma_{y}^{2}(h)=E\left((y(t+h)-y(t))^{2}\right)$, the process $Z$ satisfies the condition (A). Then it follows from Corollary 3.2 that for an arbitrary $\varepsilon^{\prime \prime}>0$ and for almost all $\omega$ there exists an

$\left({ }^{17}\right)$ See Remark 3 in $\S 2$. 
$h_{0}(\omega)$ such that $0<h<h_{0}(\omega)$ implies

$$
\begin{aligned}
\sup \{|z(t+h, \omega)-z(t, \omega)| ; 0 & \leqq t \leqq 1-h\}<\sigma_{z}(h)\left\{\left(2+\varepsilon^{\prime \prime}\right) \log (1 / h)\right\}^{1 / 2} \\
& \leqq\left\{\frac{1+\varepsilon^{\prime 2} C_{26} / C_{4}}{1+\varepsilon^{\prime 2}}\right\}^{1 / 2}\left\{\frac{\left(2+\varepsilon^{\prime \prime}\right) C_{4} h^{\alpha}}{(\log (1 / h))^{\beta-1}}\right\}^{1 / 2}
\end{aligned}
$$

and

$$
\sup \{|y(t+h)-y(t)| ; 0 \leqq t \leqq 1-h\}<\left\{\left(2+\varepsilon^{\prime \prime}\right) C_{4} \frac{h^{\alpha}}{(\log (1 / h))^{\beta-1}}\right\}^{1 / 2}
$$

So we have for $h<h_{0}(\omega)$

$$
\begin{aligned}
\sup \{\mid x(t+h)- & x(t) \mid ; 0 \leqq t \leqq 1-h\} \\
\leqq & \left(1+\varepsilon^{\prime 2}\right)^{1 / 2} \sup \{|z(t+h)-z(t)| ; 0 \leqq t \leqq 1-h\} \\
& +\varepsilon^{\prime} \sup \{|y(t+h)-y(t)| ; 0 \leqq t \leqq 1-h\} \\
\leqq & \left\{C_{4} \frac{h^{\alpha}}{(\log (1 / h))^{\beta-1}}\right\}^{1 / 2}\left\{\left(1+\varepsilon^{\prime 2} C_{26} / C_{4}\right)^{1 / 2}+\varepsilon^{\prime}\right\}\left(2+\varepsilon^{\prime \prime}\right)^{1 / 2}
\end{aligned}
$$

For any $\varepsilon>0$, if we take $\varepsilon^{\prime}$ and $\varepsilon^{\prime \prime}$ such that

$$
\left\{\left(1+\varepsilon^{\prime 2} C_{26} / C_{4}\right)^{1 / 2}+\varepsilon^{\prime}\right\}^{2}\left(2+\varepsilon^{\prime \prime}\right)<(2+\varepsilon),
$$

then (73) proves Theorem 6. Q.E.D.

As an application of Theorem 6, we can prove Corollary 6.1.

Proof of Corollary 6.1. According to Theorem 6, it suffices to show that the relation

$$
\int_{0}^{\infty} x^{\alpha}|\log (1+x)|^{\beta} d F(x)<\infty
$$

implies

$$
\sigma^{2}(h)=o\left(h^{\alpha} /(\log (1 / h))^{\beta}\right)\left({ }^{18}\right) .
$$

To show this we first consider the function

$$
g(h ; x)=\frac{(\log (1 / h))^{\beta}}{h^{\alpha}}(1-\cos h x) .
$$

Let $\delta(<1)$ be a positive number such that $x^{-\alpha}|\log x|^{\beta}$ is monotone decreasing in $x \in(0, \delta)$ and $x^{2-\alpha}|\log x|^{\beta}$ is monotone increasing in $x \in(0, \delta)$. Then, for $0<h<\delta$, it follows that for $x \geqq 1 / h$

$$
g(h, x) \leqq 2 h^{-\alpha}|\log h|^{\beta} \leqq 2 x^{\alpha}(\log x)^{\beta},
$$

and for $\delta<x<1 / h$

$$
\begin{aligned}
g(h ; x) & \leqq \frac{(\log (1 / h))^{\beta}}{h^{\alpha}} \frac{h^{2} x^{2}}{2}=h^{2-\alpha}|\log h|^{\beta} x^{2} / 2 \\
& \leqq x^{\alpha}|\log x|^{\beta} / 2
\end{aligned}
$$

$\left({ }^{18}\right) f(x)=o(g(x))$ expresses $\lim _{x \rightarrow 0} f(x) / g(x)=0$. 
So we have $g(h ; x) \leqq 2 x^{\alpha}|\log x|^{\beta}, \delta<x<\infty$.

Now (74) shows that $x^{\alpha}|\log x|^{\beta}$ is integrable with respect to the measure $d F$. So we have

$$
\lim _{h \rightarrow 0} \frac{\sigma^{2}(h)(\log (1 / h))^{\beta}}{h^{\alpha}}=\lim _{h \rightarrow 0} 2 \int_{0}^{\infty} g(h ; x) d F(x)=0,
$$

as was to be proved. Q.E.D.

\title{
REFERENCES
}

1. Ju. K. Beljaev, Continuity and Hölder's conditions for sample functions of stationary Gaussian processes, Proc. Fourth Berkeley Sympos. Math. Stat. and Prob., vol. II, Univ. of California Press, Berkeley, Calif., 1961, pp. 23-33. MR 26 \#815.

2. K. L. Chung and P. Erdös, On the application of the Borel-Cantelli lemma, Trans. Amer. Math. Soc. 72 (1952), 179-186. MR 13, 567.

3. K. L. Chung, P. Erdös and T. Sirao, On the Lipschitz's condition for Brownian motion, J. Math. Soc. Japan 11 (1959), 263-274. MR 22 \#12602.

4. X. Fernique, Continuité des processus Gaussiens, C. R. Acad. Sci. Paris 258 (1964), 6058-6060. MR 29 \#1662.

5. G. A. Hunt, Random Fourier transforms, Trans. Amer. Math. Soc. 71 (1951), 38-69. MR 14, 465.

6. M. Loève, Probability theory, 2nd ed., The University Series in Higher Math., Van Nostrand, Princeton, N. J., 1960. MR 23 \#A670.

7. T. Sirao, On the continuity of Brownian motion with a multidimensional parameter, Nagoya Math. J. 16 (1960), 135-156. MR 22 \#8570.

8. T. Sirao and H. Watanabe, On the Hölder continuity of stationary Gaussian processes, Proc. Japan Acad. 44 (1968), 482-484.

\author{
NAGOYA UNIVERSITY, \\ NAGOYA, JAPAN \\ ROCKEFELLER UNIVERSITY, \\ NeW York, NeW YorK \\ KYUSHU UNIVERSITY, \\ FUKUOKa City, JAPAN
}

